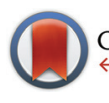

CrossMark \& click for updates

Cite this: Dalton Trans., 2016, 45 2148

Received 11th September 2015, Accepted 29th October 2015

DOI: $10.1039 / c 5 d t 03558 a$

www.rsc.org/dalton

\title{
Synthesis of 3-stannyl and 3-silyl propargyl phosphanes and the formation of a phosphinoallene $\uparrow$
}

\author{
Amy J. Saunders and Ian R. Crossley*
}

\begin{abstract}
The group 14 chloropropargyls $\mathrm{R}_{3} \mathrm{EC} \equiv \mathrm{CCCH}_{2} \mathrm{Cl}\left(\mathrm{R}_{3} \mathrm{E}={ }^{n} \mathrm{Bu}_{3} \mathrm{Sn}, \mathrm{Ph}_{3} \mathrm{Sn}, \mathrm{Me}_{2} \mathrm{PhSi},{ }^{\mathrm{i}} \mathrm{Pr}_{3} \mathrm{Si},{ }^{n} \mathrm{Pr}_{3} \mathrm{Si},{ }^{n} \mathrm{Bu}_{3} \mathrm{Si}\right)$, obtained by a modified literature procedure, react with $\mathrm{LiPPh}_{2}$ to afford the novel propargyl phosphanes $\mathrm{Ph}_{2} \mathrm{PCH}_{2} \mathrm{C} \equiv \mathrm{CER}_{3}$ in high yield, as viscous oils; $\left(\mathrm{Me}_{3} \mathrm{Si}_{2} \mathrm{PCH}_{2} \mathrm{C} \equiv \mathrm{CSiPhMe}_{2}\right.$ is similarly obtained from LiP$\left(\mathrm{SiMe}_{3}\right)_{2}$. In contrast, the reaction of $\mathrm{PhC} \equiv \mathrm{CCH}_{2} \mathrm{MgCl}$ with $\mathrm{CIP}\left(\mathrm{NEt}_{2}\right)_{2}$ fails to produce a comparable propargyl phosphane, but generates preferentially $(>70 \%)$ the novel phosphinoallene $\left(\mathrm{Et}_{2} \mathrm{~N}\right)_{2} \mathrm{PC}(\mathrm{Ph})=\mathrm{C}=\mathrm{CH}_{2}$, which is characterised spectroscopically, and through its reaction with $\mathrm{HCl}$. The coordination chemistry of representative phosphanes is explored with respect to platinum and palladium for the first time.
\end{abstract}

\section{Introduction}

Tertiary phosphanes are both ubiquitous and innumerable, being the subject of exhaustive efforts to control steric and electronic profiles through substituent modification, driven by their utility as ligands. The opportunities to impose steric bulk and/or asymmetry within the metal coordination sphere offer particular impetus, typically directed toward symmetric $\mathrm{R}_{3} \mathrm{P}$ and chiral $\mathrm{PRR}^{\prime} \mathrm{R}^{\prime \prime}$ derivatives respectively. Equally important are derivatives of the type $\mathrm{R}_{2} \mathrm{PR}^{\prime}$ ( $\mathrm{R}=$ aryl, alkyl) that occupy the intermediate ground, allowing for subtle variation of sterics and electronics (variation of $\mathrm{R}^{\prime}$ ), while also imposing some level of asymmetry about the metal. Moreover, the ready availability of $\mathrm{R}_{2} \mathrm{PX}(\mathrm{X}=$ halide, $\mathrm{H}$ ) renders a convenient scaffold with which to investigate more elaborate and functional substituents $\left(\mathrm{R}^{\prime}\right)$.

Despite prolific levels of activity in phosphane synthesis, ${ }^{1}$ particularly systems of the type $\mathrm{R}_{2} \mathrm{PR}^{\prime}$, surprising oversights remain, a case in point being the dearth of systems bearing a propargylic substituent (viz. $\mathrm{CH}_{2} \mathrm{C} \equiv \mathrm{CR}^{\prime}$ ). Indeed, while alkynyl phosphanes are common, ${ }^{2}$ their propargyl counterparts are limited to $\mathrm{R}_{2} \mathrm{PCH}_{2} \mathrm{C} \equiv \mathrm{CR}^{\prime}\left(\mathrm{R}=\mathrm{Ph}, \mathrm{R}^{\prime}=\mathrm{H},{ }^{3} \mathrm{Me},{ }^{4} \mathrm{Ph} ;{ }^{5} \mathrm{R}=\mathrm{Cy}\right.$, $\left.{ }^{\mathrm{i}} \mathrm{Pr}, \mathrm{R}^{\prime}=\mathrm{H}, \mathrm{SiMe}_{3} ;^{6}\right)$, typically isolated as stabilised $\mathrm{BH}_{3}$ adducts, $\mathrm{R}_{2} \mathrm{PCH}\left\{\mathrm{OSiMe}_{3}\right\} \mathrm{C} \equiv \mathrm{CPh}(\mathrm{R}=\mathrm{Ph}, \mathrm{Et}){ }^{7}\left\{\left(\mathrm{Me}_{3} \mathrm{Si}\right)_{2} \mathrm{~N}\right\}-$ $\mathrm{RPCH}_{2} \mathrm{C} \equiv \mathrm{CSiMe}_{3} \quad\left(\mathrm{R}=\mathrm{Ph},{ }^{8} \mathrm{Et},{ }^{8} \mathrm{Cl}^{9}\right)$, the diphosphane $\mathrm{Ph}_{2} \mathrm{PCH}_{2} \mathrm{C} \equiv \mathrm{CCH}_{2} \mathrm{PPh}_{2},{ }^{10}$ and the bis-propargylphosphanes $\mathrm{RP}\left\{\mathrm{CH}_{2} \mathrm{C} \equiv \mathrm{CR}^{\prime}\right\}_{2}\left(\mathrm{R}=\mathrm{Np}, \mathrm{R}^{\prime}=\mathrm{H}, \mathrm{SiMe}_{3} ;{ }^{11} \mathrm{R}=\mathrm{CH}_{2} \mathrm{CMe}_{2} \mathrm{Et}, \mathrm{R}^{\prime}=\right.$

Department of Chemistry, University of Sussex, Brighton, UK.

E-mail: i.crossley@sussex.ac.uk; Fax: +44 (0)1273 876687; Tel: +44 (0)1273 877302 $\dagger$ Electronic supplementary information (ESI) available: Representative NMR spectra for compounds 8, 15 and 16, in lieu of bulk elemental analysis data. See DOI: $10.1039 / \mathrm{c} 5 \mathrm{dt} 03558 \mathrm{a}$
$\left.\mathrm{H} ;{ }^{11} \mathrm{R}=\mathrm{Ph}, \mathrm{R}^{\prime}={ }^{n} \mathrm{Bu} ;{ }^{12} \mathrm{R}=\mathrm{Mes}, \mathrm{R}^{\prime}=\mathrm{SiMe}_{3}{ }^{13}\right)$, which are typically putative intermediates in the synthesis of macrocycles. The primary propargyl phosphane $\mathrm{H}_{2} \mathrm{PCH}_{2} \mathrm{C} \equiv \mathrm{CH}$ has also been reported. ${ }^{14}$

This lack of activity is surprising given continued interest in developing polyfunctional phosphorus-containing molecules, driven by their utility as ligands, optoelectronically active $\pi$-conjugates ${ }^{15}$ and, topically, frustrated Lewis pairs (FLPs). ${ }^{16}$ In these contexts, propargylphosphanes should constitute ideal 'building-block' substrates, and allow for incorporation of further functionality (e.g. by cycloaddition, hydroboration, hydrophosphination) akin to their more extensively utilised alkynyl, alkenyl and allyl counterparts. Moreover, they embody intrinsic potential to act as $\sigma / \pi$-chelating ligands. Indeed, among very limited coordination chemistry reported to date, the $\mu-(\sigma-P, \pi-C \equiv C)$ bridging mode has been described for $\left[\mathrm{Cp}_{2} \mathrm{Rh}_{2}(\mathrm{CO})\left(\mu-\eta^{1}: \eta^{1}-\mathrm{CF}_{3} \mathrm{C}_{2} \mathrm{CF}_{3}\right)\left\{\mathrm{PPh}_{2} \mathrm{CH}_{2} \mathrm{C} \equiv \mathrm{CMe} \mathrm{CO}_{2}(\mathrm{CO})_{6}\right]\right.$, obtained by reaction of $\left[\mathrm{Co}_{2}(\mathrm{CO})_{8}\right]$ with the dirhodium complex $\left[\mathrm{Cp}_{2} \mathrm{Rh}_{2}(\mathrm{CO})\left(\mu-\eta^{1}: \eta^{1}-\mathrm{CF}_{3} \mathrm{C}_{2} \mathrm{CF}_{3}\right)\left\{\mathrm{PPh}_{2} \mathrm{CH}_{2} \mathrm{C} \equiv \mathrm{CMe}\right\}\right]$.

The remaining complexes described to date involve monodentate coordination of the phosphane, typically to metals of the mid transition series, with saturated coordination spheres. Thus, $\left[\mathrm{M}(\mathrm{CO})_{5}\left(\mathrm{PR}_{2} \mathrm{C} \equiv \mathrm{CH}\right)\right]\left(\mathrm{M}=\mathrm{Mo}, \mathrm{R}=\mathrm{Ph},{ }^{17} \mathrm{DBP} ;{ }^{18}+\mathrm{Cr},{ }^{19} \mathrm{R}=\right.$ $\left.\mathrm{Ph}, \mathrm{SiMe}_{3}\right),\left[\mathrm{Mo}(\mathrm{CO})_{3}\left(\mathrm{PH}_{2} \mathrm{CH}_{2} \mathrm{C} \equiv \mathrm{CH}\right)_{3}\right],\left[\mathrm{Mo}(\mathrm{CO})_{4}\left(\mathrm{PH}_{2} \mathrm{C} \equiv \mathrm{CH}\right)^{20}\right]$ and $\left[\mathrm{Cp}^{\mathrm{R}} \mathrm{Mn}(\mathrm{CO})_{2}\left(\mathrm{PPh}_{3-n}\left(\mathrm{CH}_{2} \mathrm{C} \equiv \mathrm{CH}\right)_{n}\right]\left(\mathrm{Cp}^{\mathrm{R}}=\mathrm{Cp}, n=1,2 ; \mathrm{Cp}^{\mathrm{R}}\right.\right.$ $\left.=\mathrm{Cp}^{\mathrm{Me}}, n=1\right) .{ }^{19}$ have been obtained directly from the respective phosphanes and suitable metal salts, as has the bimetallic complex $\left[\left\{\mathrm{HC} \equiv \mathrm{CCH}_{2} \mathrm{Ph}_{2} \mathrm{P}\right\} \mathrm{Ru}(\mathrm{CO})_{3}\left(\mu-\mathrm{PPh}_{2}\right) \mathrm{Co}(\mathrm{CO})_{3}\right]{ }^{21}$ In contrast, $\quad\left[\mathrm{Co}(\mathrm{NO})(\mathrm{CO})\left(\mathrm{PPh}_{2} \mathrm{CH}_{2} \mathrm{C} \equiv \mathrm{CH}\right)_{2}\right]^{22}$ and the ruthenium phthalocyaninato $\left(\mathrm{Pc}^{2-}\right)$ complex $\left[\mathrm{Ru}(\mathrm{Pc})\left(\mathrm{PPh}_{2} \mathrm{CH}_{2} \mathrm{C} \equiv \mathrm{CCH}_{3}\right)_{2}\right]^{23}$

$\ddagger$ DBP = dibenzophosphole. 
are obtained from the respective diphenylphosphane complexes, via in situ deprotonation (BuLi) and quenching with the appropriate propargylic bromide; [CpMo $\left\{\kappa^{2}-O, P-\mathrm{P}(\mathrm{O}) \mathrm{Mes}^{*}\right.$ $\left.\left.\left(\mathrm{CH}_{2} \mathrm{C} \equiv \mathrm{CH}\right)\right\}\right]$ is similarly prepared, but without need for base. $^{24}$ Finally, $\left[\mathrm{W}(\mathrm{CO})_{5}\left\{\mathrm{PPh}(\mathrm{OMe}) \mathrm{C}(\mathrm{H}) \mathrm{Me}\left(\mathrm{C} \equiv \mathrm{CSi}^{\mathrm{i}} \mathrm{Pr}_{3}\right)\right\}\right]$ was obtained upon methanolysis of the putative phosphaalkene $\left[\mathrm{W}(\mathrm{CO})_{5}\left\{\mathrm{P}(\mathrm{Ph})=\mathrm{CMe}\left(\mathrm{C} \equiv \mathrm{CSi}^{\mathrm{i}} \mathrm{Pr}_{3}\right)\right\}\right] .^{25}$

Notably, no complexes of the group 10 metals have been described, though the formally related diphosphane-bridged complexes $\quad\left[\mathrm{L}_{n} \mathrm{M}\left\{\mu-\eta^{1}: \eta^{1}-\mathrm{PPh}_{2} \mathrm{CH}_{2} \mathrm{C} \equiv \mathrm{CCH}_{2} \mathrm{PPh}_{2}\right\}\right]_{2} \quad\left(\mathrm{~L}_{n} \mathrm{M}=\right.$ $\mathrm{Cl}_{2} \mathrm{Pt},(\mathrm{OC})_{2} \mathrm{Ni}$ ) have been reported, ${ }^{26}$ alongside examples with other metals $\left(\mathrm{L}_{n} \mathrm{M}=\mathrm{AuCl}, \mathrm{CpCoI}_{2}, \mathrm{CpFe}(\mathrm{CO})_{2}{ }^{+}, \mathrm{CpFeBr}(\mathrm{CO})\right.$, $\left.\mathrm{CpMn}(\mathrm{NO})(\mathrm{CO}), \quad \mathrm{CpMo}(\mathrm{CO})_{2}\left(\mathrm{COCH}_{3}\right),{ }^{26} \mathrm{Mo}(\mathrm{CO})_{4}{ }^{27}\right)$. The intriguing tetrameric complex $\left[\left\{\eta^{2}-C, C-\mathrm{Mo}(\mathrm{CO})_{4}\left(\eta^{2}-P, P-\right.\right.\right.$ $\left.\left.\left.\mathrm{PPh}_{2} \mathrm{CH}_{2} \mathrm{C} \equiv \mathrm{CCH}_{2} \mathrm{PPh}_{3}\right)\right\}_{3} \mathrm{Mo}(\mathrm{CO})\right]$, has also been described. ${ }^{27}$

We have recently been interested in the synthesis and study of reactive and functional phosphanes ${ }^{28}$ and organometallic phosphacarbons, ${ }^{29}$ with the goal of developing novel ambiphilic systems ${ }^{28 c}$ and molecular conductive and/or optoelectronically active molecules. ${ }^{29 b}$ In continuing these works, we have had cause to access propargyl phosphanes of the type $\mathrm{R}_{2} \mathrm{P}$ $\left(\mathrm{CH}_{2} \mathrm{C} \equiv \mathrm{CER}_{3}^{\prime}\right)(\mathrm{E}=\mathrm{Si}, \mathrm{Sn})$ as intermediates, seeking to exploit their capacity for desilylative/destannylative functionalisation. In view of the limited range of propargyl phosphanes reported previously, we thus undertook to prepare a putative series of such materials; viz. $\mathrm{Ph}_{2} \mathrm{P}\left(\mathrm{CH}_{2} \mathrm{C} \equiv \mathrm{CER}_{3}\right)$ ( $\mathrm{E}=\mathrm{Si}$, Sn), which we describe herein, along with attempts to obtain ' $\left(\mathrm{Et}_{2} \mathrm{~N}\right)_{2} \mathrm{P}$ ' derivatives, leading to the generation of a novel, and very rare, phosphinoallene. We also outline the coordination chemistry of representative propargylphosphanes toward $\mathrm{Pd}$ and Pt, reporting the first such complexes from group 10, and the first to involve coordinately unsaturated metal centres.

\section{Results and discussion}

\section{Phosphane synthesis}

The silyl and stannyl chloropropargyl precursors $\mathrm{R}_{3} \mathrm{EC} \equiv \mathrm{CCH}_{2} \mathrm{Cl}$ were prepared following a modified literature procedure (Scheme 1), ${ }^{30}$ via the low-temperature $\left(\begin{array}{ll}-78 & { }^{\circ} \mathrm{C}\end{array}\right)$ lithiation of $\mathrm{HC} \equiv \mathrm{CCH}_{2} \mathrm{Cl}$, quenched with $\mathrm{R}_{3} \mathrm{SnCl}$ (1 and 2) or $\mathrm{R}_{3} \mathrm{SiCl}(3-7)$. The silanes were amenable to purification by

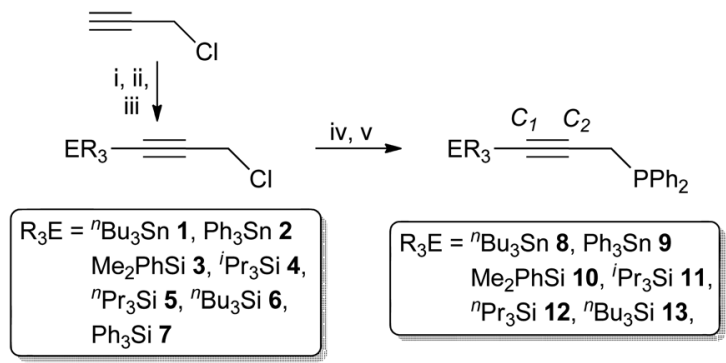

Scheme 1 Reagents and conditions: (i) ${ }^{n} \mathrm{BuLi},-78^{\circ} \mathrm{C}, \mathrm{Et}_{2} \mathrm{O}, 30 \mathrm{~min}$; (ii) $\mathrm{R}_{3} \mathrm{ECl},-78{ }^{\circ} \mathrm{C}, 30 \mathrm{~min}$; (iii) warm to ambient, stir $18 \mathrm{~h}$; (iv) $\mathrm{LiPPh}_{2}$, $-78^{\circ} \mathrm{C}, \mathrm{Et}_{2} \mathrm{O}, 30 \mathrm{~min}$; (v) r.t. $18 \mathrm{~h}$. reduced-pressure distillation, apart from the solid $7(\mathrm{R}=\mathrm{Ph})$, which was sublimed. However, both silanes and stannanes are typically obtained in adequate purity for further reaction (>95\%) upon extraction with pentane. In each case, compound identity was apparent from the ${ }^{1} \mathrm{H}$ NMR spectra, which exhibit resonances associated with the group 14 fragment, integrating consistently against that of the propargyl methylene moiety $\left(\delta_{\mathrm{H}}\right.$ 3.5-3.7), which is shifted by $c a$. $0.3 \mathrm{ppm}$ to lower frequency compared with propargyl chloride. Moreover, correlations are observed between the methylenic resonances and respective group 14 centre in each case $\left({ }^{1} \mathrm{H}-\mathrm{X} \mathrm{HMBC} ; \mathrm{X}={ }^{119} \mathrm{Sn},{ }^{29} \mathrm{Si}\right)$; for the stannanes the ${ }^{4} J_{\mathrm{SnH}}$ coupling $(\sim 10 \mathrm{~Hz})$ is also large enough to resolve tin satellites. The ${ }^{13} \mathrm{C}\left\{{ }^{1} \mathrm{H}\right\}$-NMR data are similarly consistent, while bulk purity was confirmed from microanalytical data. It is noted that $\mathbf{1},{ }^{31} 2,,^{30,31} \mathbf{3}^{32}$ and $\mathbf{4}^{33}$ have been previously obtained via alternate methodology.

Ethereal solutions of $\mathbf{1}$ to 6 were added $\left(-78{ }^{\circ} \mathrm{C}\right)$ to $\mathrm{LiPPh}_{2}$ in ether (formed by in situ lithiation of $\mathrm{HPPh}_{2}$ with ${ }^{n} \mathrm{BuLi}$ ) and the mixtures stirred overnight to afford the propargyl phosphanes $\mathrm{Ph}_{2} \mathrm{P}\left(\mathrm{CH}_{2} \mathrm{C} \equiv \mathrm{CER}_{3}\right)$ (8-13, Scheme 1). Extraction with pentane afforded the phosphanes as viscous oils, the silyl derivatives 10-13 requiring no further purification. In contrast, stannanes formed in admixture with ${ }^{n} \mathrm{Bu}_{4} \mathrm{Sn}$ (1:4 of 8) or ${ }^{n} \mathrm{BuPh}_{3} \mathrm{Sn}$ (1:1 with 9), presumably due to metathesis of 1 and 2 with residual ${ }^{n} \mathrm{BuLi}$, as is common among Sn(Iv) organyls. ${ }^{34}$ Both 8 and $\mathbf{9}$ are unstable toward distillation and were thus only characterised spectroscopically, though for $\mathbf{8}$, further data were obtained by coordination to platinum (vide infra), which proceeds cleanly. In contrast, 9 forms in a complex, inseparable mixture that includes unidentified by-products; it has not been studied further.

Compounds $\mathbf{8}$ to $\mathbf{1 3}$ are identified from characteristic spectroscopic data (Table 1), the alkynic moieties exhibiting marginal change from those of the parent propargyls. Retention of the group 14 fragments is universally apparent $\left({ }^{1} \mathrm{H}-\mathrm{X} \mathrm{HMBC}\right)$, with 8 and 9 also allowing for resolution of ${ }^{119} \mathrm{Sn}$ satellites $\left({ }^{4} J_{\mathrm{SnP}} \sim 14 \mathrm{~Hz}\right)$ in the ${ }^{31} \mathrm{P}\left\{{ }^{1} \mathrm{H}\right\}$ spectra. The ${ }^{119} \mathrm{Sn}$ spectra of 8 and 9 indicate the presence of ${ }^{n} \mathrm{Bu}_{4} \mathrm{Sn}\left(\delta_{\mathrm{Sn}}-12.0\right)^{35}$ and ${ }^{n} \mathrm{BuPh}_{3} \mathrm{Sn}\left(\delta_{\mathrm{Sn}}-98.3\right)^{36}$ by-products respectively.

Attempts to vary the nature of the phosphanyl substituents met with limited success. Dicyclohexyl analogues failed to form, regenerating $\mathrm{HPCy}_{2}$ as the only phosphorus-containing product, which presumably reflects the greater basicity and steric bulk of ' $\mathrm{PCy}_{2}$ ' $\left(c f\right.$. ' $\mathrm{PPh}_{2}$ '), favouring proton-abstraction

Table 1 Selected NMR spectroscopic data for proparylphosphanes $8-14^{a}$

\begin{tabular}{llllll}
\hline & $\delta_{\mathrm{P}}$ & $\delta_{\mathrm{H}}\left(\mathrm{CH}_{2}\right)\left[J_{\mathrm{PH}}\right]^{b}$ & $\delta_{\mathrm{C}}\left(\mathrm{C}_{1}\right)$ & $\delta_{\mathrm{C}}\left(\mathrm{C}_{2}\right)$ & $\delta_{\mathrm{E}}(\mathrm{E})$ \\
\hline $\mathbf{8}$ & -13.4 & $2.87[1.6]$ & 85.0 & 107.0 & $-68.4\left[{ }^{119} \mathrm{Sn}\right]$ \\
$\mathbf{9}$ & -13.2 & $2.84[3.0]$ & 82.8 & 109.0 & $-168.4\left[{ }^{119} \mathrm{Sn}\right]$ \\
$\mathbf{1 0}$ & -13.5 & $2.76[2.9]$ & 84.7 & 105.0 & $-22.9\left[{ }^{29} \mathrm{Si}\right]$ \\
$\mathbf{1 1}$ & -13.5 & $2.75[2.3]$ & 83.3 & 105.0 & $-3.03\left[{ }^{29} \mathrm{Si}\right]$ \\
$\mathbf{1 2}$ & -13.6 & $2.76[2.5]$ & 85.4 & 103.0 & $-14.5\left[{ }^{29} \mathrm{Si}\right]$ \\
$\mathbf{1 3}$ & -13.5 & $2.76[2.3]$ & 85.5 & 104.0 & $-12.9\left[{ }^{29} \mathrm{Si}\right]$ \\
$\mathbf{1 4}$ & -158.9 & $2.43[1.3]$ & 83.3 & 109.3 & $-22.8 ; 3.7\left[{ }^{29} \mathrm{Si}\right]$
\end{tabular}

${ }^{a}$ As $\mathrm{C}_{6} \mathrm{D}_{6}$ solutions. ${ }^{b}$ Couplings in Hz. 
from the chloropropargyls over $\mathrm{S}_{\mathrm{N}} 2$ substitution. In contrast, reactions with $\mathrm{LiP}\left(\mathrm{SiMe}_{3}\right)_{2}$ did afford species consistent with the desired propargylphosphanes, though in admixture with several significant contaminants, which defied separation or characterisation. Nonetheless, $\mathrm{Me}_{2} \mathrm{PhSiC} \equiv \mathrm{CCH}_{2} \mathrm{P}\left(\mathrm{SiMe}_{3}\right)_{2}$ (14) was obtained as the primary product ( $92 \%$ by $\left.{ }^{31} \mathrm{P}\left\{{ }^{1} \mathrm{H}\right\}-\mathrm{NMR}\right)$ in admixture with $\mathrm{P}\left(\mathrm{SiMe}_{3}\right)_{3}(4 \%)$ and a mono-silylphosphane $\left(\delta_{\mathrm{P}}\right.$ $-84.4 ; 4 \%$ ), which presumably result from disproportionation; indeed, the bulk composition is consistent with that of $\mathbf{1 4}$.

Given these difficulties, the generation of propargyl Grignard reagents from 1 to 7 was considered as an alternative approach; however, these reactions proved unreliable, presumably reflecting diminished reactivity of the halide in comparison to organo-propargyl derivatives. Indeed, though less favoured than their bromide analogues, propargyl chlorides have been shown to form Grignard reagents, ${ }^{37}$ and we encountered no difficulty is generating ' $\mathrm{PhC} \equiv \mathrm{CCH}_{2} \mathrm{MgCl}$ ' under comparable conditions. However, our efforts to quench this reagent with $\left(\mathrm{Et}_{2} \mathrm{~N}\right)_{2} \mathrm{PCl}$ led to an unexpected outcome.

\section{Formation of a phosphino-allene}

The addition of freshly prepared ' $\mathrm{PhC} \equiv \mathrm{CCH}_{2} \mathrm{MgCl}$ ' to a cooled $\left(-78^{\circ} \mathrm{C}\right)$ THF solution of $\left(\mathrm{Et}_{2} \mathrm{~N}\right)_{2} \mathrm{PCl}$ affords, after workup, a deep red oil comprising one predominant phosphoruscontaining product $(\mathbf{1 5} ; \mathbf{7 5} \%)$. The spectroscopic features of $\mathbf{1 5}$ confirm the presence of a ' $\left(\mathrm{Et}_{2} \mathrm{~N}\right)_{2} \mathrm{P}$ ' moiety $\left(\delta_{\mathrm{P}} 90.9 ; c f\right.$. PhP$\left.\left(\mathrm{NEt}_{2}\right)_{2} 97.9,{ }^{38} \mathrm{H}_{2} \mathrm{C}=\mathrm{C}(\mathrm{H})-\mathrm{P}\left(\mathrm{NEt}_{2}\right)_{2} 89.9{ }^{39}\right)$, the ${ }^{1} \mathrm{H}$ NMR resonances integrating consistently against those for single equivalences of aromatic and methylenic fragments. However, the methylenic moiety is significantly deshielded $\left(\delta_{\mathrm{H}} 4.72 . \delta_{\mathrm{C}} 75.0\right)$ relative to both $\mathrm{PhC} \equiv \mathrm{CCH}_{2} \mathrm{Cl}\left(\delta_{\mathrm{H}} 4.39, \delta_{\mathrm{C}} 31.2\right)$ and propargyl phosphanes, and exhibits appreciably greater magnitude coupling to phosphorus $\left(\left|J_{\mathrm{PH}}\right|=7.1 \mathrm{~Hz}\right)$ than 8-14. The unsaturated carbon centres are also heavily deshielded $\left(\delta_{\mathrm{c}} 137.4\left(J_{\mathrm{PC}} 19.0\right.\right.$ $\left.\mathrm{Hz}) \mathrm{C}_{1} ; 209.9\left(J_{\mathrm{PC}} 11.3 \mathrm{~Hz}\right) \mathrm{C}_{2}\right)$, the latter in particular being more characteristic of an allenic, ${ }^{40}$ rather than alkynic centre; indeed, these data are in good agreement with those for the limited range of phosphinoallenes $\S$ (Table 2) described previously. ${ }^{14,41}$ We thus confidently formulate 15 as $\left(\mathrm{Et}_{2} \mathrm{~N}\right)_{2} \mathrm{PC}$ $(\mathrm{Ph})=\mathrm{C}=\mathrm{CH}_{2}$ (Scheme 2).

The reaction of propargyl Grignard reagents with $\mathrm{R}_{2} \mathrm{PCl}$ has been noted to afford mixtures that include allenyl-phosphanes, their proportion being dependent on the nature of ' $R$ '. ${ }^{42}$ However, this is to our knowledge the first example of an allenylphosphane being obtained as the major product $(>70 \%)$ in such a reaction, with minimal levels $(<2 \%)$ of the propargyl tautomer. While we have not further studied this reaction, the noted outcome might reasonably be considered to reflect either enhanced stability of the $\alpha$-phenyl-allenyl carbanion over its propargylic counterpart (localisation at an $\mathrm{sp}^{2}$, rather than $\mathrm{sp}^{3}$ centre) or be the result of conjugate addition, favoured by the relatively 'soft' $\mathrm{ClP}\left(\mathrm{NEt}_{2}\right)_{2}$ electrophile, as com-

$\S$ We note that allenylphosphonates have been more heavily studied; indeed, several of the limited allenylphosphines reported previously have been obtained through reduction of the respective phosphonates.
Table 2 Selected ${ }^{1} \mathrm{H}$ and ${ }^{13} \mathrm{C}\left\{{ }^{1} \mathrm{H}\right\}$-NMR spectroscopic data for precedent phosphinoallenes ${ }^{a, b}$

\begin{tabular}{llll}
\hline & $\delta_{\mathrm{H}}\left(=\mathrm{CH}_{2}\right)$ & $\delta_{\mathrm{C}}\left(=\mathrm{CH}_{2}\right)$ & $\delta_{\mathrm{C}}(=\mathrm{C}=)$ \\
\hline $\mathrm{Mes}(\mathrm{H}) \mathrm{PC}(\mathrm{Me})=\mathrm{C}=\mathrm{CH}_{2}$ & 4.40 & 71.12 & 208.0 \\
$\mathrm{Mes}(\mathrm{Me}) \mathrm{PC}(\mathrm{Me})=\mathrm{C}=\mathrm{CH}_{2}$ & 4.64 & 73.26 & 204.9 \\
$\mathrm{Mes}\left(\mathrm{Me} \mathrm{CH}_{3} \mathrm{Si}\right) \mathrm{PC}(\mathrm{Me})=\mathrm{C}=\mathrm{CH}_{2}$ & 4.55 & 72.35 & 206.4 \\
$\mathrm{Mes}(\mathrm{Cl}) \mathrm{PC}(\mathrm{Me})=\mathrm{C}=\mathrm{CH}_{2}$ & 4.57 & 74.65 & 205.5 \\
$\mathrm{Ph}_{2} \mathrm{PC}(\mathrm{H})=\mathrm{C}=\mathrm{CH}_{2}$ & - & 71.7 & 213.2 \\
$\mathrm{Ph}_{2} \mathrm{PC}(\mathrm{Me})=\mathrm{C}=\mathrm{CH}_{2}$ & - & 70.7 & 210.3 \\
$\mathrm{Ph}_{2} \mathrm{PC}(\mathrm{H})=\mathrm{C}=\mathrm{C}(\mathrm{Me})_{2}$ & - & - & 209.6 \\
${ }^{a}$ Chemical shifts in ppm. & ${ }^{b}$ Data sourced from ref. 14 and 41.
\end{tabular}

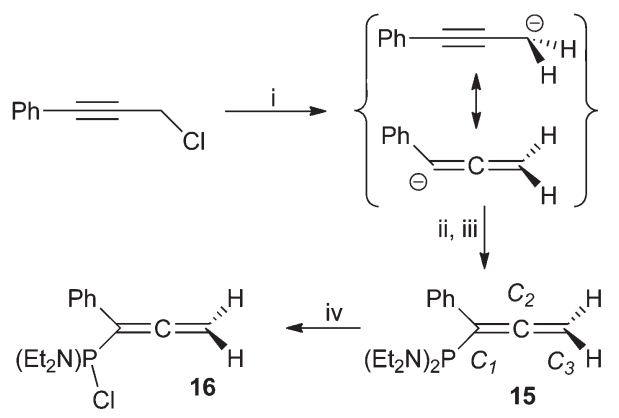

Scheme 2 Reagents and conditions: (i) $\mathrm{Et}_{2} \mathrm{O}, \mathrm{HgCl}_{2}$ (5 mol\%), $\mathrm{Mg}, \triangle$, $4 \mathrm{~h}$; (ii) $\left(\mathrm{Et}_{2} \mathrm{~N}\right)_{2} \mathrm{PCl},-78{ }^{\circ} \mathrm{C}, 30 \mathrm{~min}$; (iii) r.t. $18 \mathrm{~h}$; (iv) 2 equiv. $\mathrm{HCl} / \mathrm{Et}_{2} \mathrm{O}$.

pared, for instance, with the notionally 'harder' $\mathrm{PCl}_{3}$, with which we encountered significantly greater complexity, yielding a largely intractable mixture.

In order to confirm or dismiss the presence of $\mathrm{Cl}_{2} \mathrm{PC}(\mathrm{Ph})=$ $\mathrm{C}=\mathrm{CH}_{2}$ within this mixture, we sought to prepare an authentic sample, treating 15 with $\mathrm{HCl}$ (2 equiv.). This effected quantitative conversion to $\left(\mathrm{Et}_{2} \mathrm{~N}\right)(\mathrm{Cl}) \mathrm{PC}(\mathrm{Ph})=\mathrm{C}=\mathrm{CH}_{2}$ (16), as evidenced by the ${ }^{1} \mathrm{H}$ NMR spectrum, which indicates loss of one diethylamino moiety $\left(\mathrm{Et}_{2} \mathrm{~N} v\right.$ s. Ph resonances) and emergence of diasterotopicity for the methylenic ' $=\mathrm{CH}_{2}$ '. The phosphorus resonance of $\mathbf{1 6}$ is appreciably deshielded from that of 15, consistent with replacement of $\mathrm{NEt}_{2}$ by $\mathrm{Cl}\left(\delta_{\mathrm{P}} 122 ; c f . \mathrm{Ph}(\mathrm{Cl}) \mathrm{PNEt}_{2}\right.$ $\left.142.1^{43}\right)$. Upon further treatment with $\mathrm{HCl}$ there is superficial evidence for removal of the remaining diethylamino moiety, viz. loss of its ${ }^{1} \mathrm{H}$ NMR resonances, and of diasterotopicity of the ' $=\mathrm{CH}_{2}$ ' protons $\left(\delta_{\mathrm{H}} 4.63, \mathrm{~d}, J_{\mathrm{PH}} 3 \mathrm{~Hz}\right.$ ). However, the ${ }^{31} \mathrm{P}$ shift $\left(\delta_{\mathrm{P}} 58.7, \mathrm{t}, J_{\mathrm{PH}} 3 \mathrm{~Hz}\right)$ seems inconsistent with a species of the type $\mathrm{RPCl}_{2}$; moreover, several other, unidentified, species are apparent in both the ${ }^{1} \mathrm{H}$ and ${ }^{31} \mathrm{P}-\mathrm{NMR}$ spectra, precluding confident assignment of the bulk product.

\section{Coordination chemistry of propargylphosphanes}

As previously noted (vide supra) the coordination chemistry of propargylphosphanes is significantly underdeveloped and focused exclusively on coordinately saturated, mid-transition metals. We thus sought to prepare representative complexes featuring the unsaturated group 10 metals Pd and Pt. 


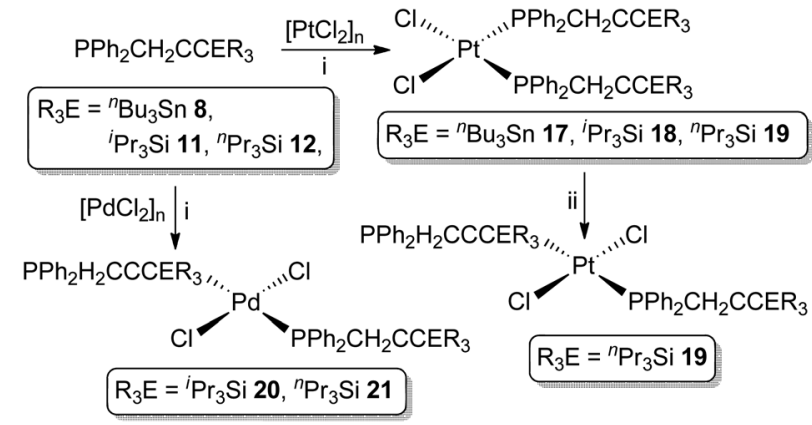

Scheme 3 Reagents and conditions: (i) $\mathrm{CH}_{2} \mathrm{Cl}_{2}, 12 \mathrm{~h}$; (ii) $\mathrm{C}_{6} \mathrm{D}_{6}, h$, $30 \mathrm{~min}$.

The propargylphosphanes $\mathbf{8 , 1 1}$ and $\mathbf{1 2}$ react with $\left[\mathrm{PtCl}_{2}\right]_{n}$, as a suspension in $\mathrm{CH}_{2} \mathrm{Cl}_{2}$, to afford exclusively the complexes cis- $\left[\mathrm{Pt}\left\{\mathrm{PPh}_{2}\left(\mathrm{CH}_{2} \mathrm{C} \equiv \mathrm{CER}_{3}\right)\right\}_{2} \mathrm{Cl}_{2}\right] \quad\left(\mathrm{ER}_{3}={ }^{n} \mathrm{Bu}_{3} \mathrm{Sn}\right.$ 17, ${ }^{\mathrm{i}} \mathrm{Pr}_{3} \mathrm{Si}$ 18, ${ }^{n} \mathrm{Pr}_{3} \mathrm{Si} 19$, Scheme 3) in excess of $75 \%$ isolated yield. For the silanes, palladium analogues $\left(\mathrm{ER}_{3}={ }^{\mathrm{i}} \mathrm{Pr}_{3} \mathrm{Si} 20,{ }^{n} \mathrm{Pr}_{3} \mathrm{Si} 21\right)$ are similarly obtained from $\left[\mathrm{PdCl}_{2}\right]_{n}$, forming exclusively as the trans isomers.

Complexes 17-21 have, thus far, not yielded X-ray quality single crystals, in common with most of the limited range of precedent examples. Nonetheless, their identities are unequivocally established from characteristic spectroscopic data, which verify the structural integrity of the ligands and coordination of the phosphorus centres $\left(\Delta \delta_{\mathrm{P}} \sim+20\right)$. For the platinum complexes 17-19, $\left|{ }^{1} J_{\mathrm{PtP}}\right|$ values of $c a .3600 \mathrm{~Hz}$ are wholly consistent with assignment of a cis geometry, while the palladium complexes exhibit virtual coupling in the ${ }^{1} \mathrm{H}$ and ${ }^{13} \mathrm{C}\left\{{ }^{1} \mathrm{H}\right\}$-NMR resonances associated with the $\mathrm{CH}_{2} \mathrm{P}$ moiety, consistent with a trans ligand arrangement. Notably, despite coordinative unsaturation of the metals, there is no evidence for either intra or intermolecular association of the pendant alkynyl moieties, the spectroscopic features of these units being little perturbed from the free ligands.

All of the complexes appear robust, both in solution and the solid state, universally resisting attempts to thermally induce cis/trans isomerisation. However, the UV irradiation (broad spectrum) of the platinum complex cis-19 over a period of 30 minutes did result in partial isomerisation, affording a mixture of cis-19 (42\%) and trans-19 (58\%). The identity of trans-19 was established on the basis of (i) reduced magnitude Pt-P coupling $\left(\left|{ }^{1} J_{\mathrm{PtP}}\right|=2601 \mathrm{~Hz}\right)$, consistent with trans- $\left[\mathrm{Pt}\left(\mathrm{PR}_{3}\right)_{2} \mathrm{X}_{2}\right]$, and (ii) manifestation of virtual coupling for the $\mathrm{CH}_{2} \mathrm{P}$ centres, as in the palladium systems. However, attempts to effect complete conversion to trans-19 through extended irradiation proved unsuccessful, no further perturbation of the isomeric distribution being achieved.

\section{Conclusions}

We have described the synthesis and characterisation of a series of novel propargylphosphanes that feature tin and silicon termini on the alkyne moiety. Attempts to increase the range of phosphanyl termini used via the reaction of $\mathrm{R}_{2} \mathrm{PCl}$ with propargyl Grignard reagents proved unsuccessful, but allowed for the generation of the novel allenylphosphine $\left(\mathrm{Et}_{2} \mathrm{~N}\right)_{2} \mathrm{PC}(\mathrm{Ph})=\mathrm{C}=\mathrm{CH}_{2}$, the first time a species of this type has been obtained as the primary product $(>70 \%)$ of such a reaction.

Representative phosphanes have been shown to form complexes $\left[\mathrm{M}\left(\mathrm{PPh}_{2} \mathrm{CH}_{2} \mathrm{C} \equiv \mathrm{CER}_{3}\right)_{2} \mathrm{Cl}_{2}\right]$ with palladium and platinum, adopting exclusively trans $(\mathrm{Pd})$ or cis $(\mathrm{Pt})$ geometries respectively, though the latter can be partially isomerised under UV irradiation. These are the first examples of propargyl phosphane complexes incorporating group 10, or indeed any unsaturated, metals and are among a very limited number $(<25)$ of coordination compounds known for any such ligands.

\section{Experimental}

\section{General methods}

All manipulations were performed under strict anaerobic conditions using standard Schlenk line and glovebox (MBraun) techniques, working under an atmosphere of dry argon or dinitrogen respectively. Solvents were distilled from appropriate drying agents and stored over either molecular sieves ( 4 A for DCM and THF) or potassium mirrors. Propargyl chloride, group 14 triorganohalides and $\mathrm{HPPh}_{2}$ were obtained from Sigma-Aldrich, purified by appropriate methods and degassed (freeze-thaw) before use. ${ }^{n} \mathrm{BuLi}$ (2.5 $\mathrm{M}$ in hexanes) was obtained from Sigma-Aldrich and titrated to establish concentration. Precious metal salts $\left(\mathrm{PtCl}_{2}, \mathrm{PdCl}_{2}\right)$ were obtained from STREM and used as supplied. $\mathrm{HP}\left(\mathrm{SiMe}_{3}\right)_{2}$ was prepared by literature procedure. ${ }^{44}$ Deuterated solvents were supplied by Goss Scientific and purified by refluxing with potassium (hydrocarbon) or $\mathrm{CaH}_{2}$ (chlorinated) for 3 days prior to use, being vacuum transferred and stored under inert atmosphere. Unless otherwise stated, NMR spectra were recorded on a Varian VNMRS $400\left({ }^{1} \mathrm{H}, 399.50 \mathrm{MHz} ;{ }^{13} \mathrm{C}, 100.46 \mathrm{MHz}\right.$; ${ }^{31} \mathrm{P}, 161.71 \mathrm{MHz} ;{ }^{29} \mathrm{Si}, 79.37 \mathrm{MHz} ;{ }^{119} \mathrm{Sn}, 148.97 \mathrm{MHz} ;{ }^{195} \mathrm{Pt}$, 85.53 MHz) or VNMRS $500\left({ }^{1} \mathrm{H} 499.91 \mathrm{MHz} ;{ }^{13} \mathrm{C}, 125.72 \mathrm{MHz}\right)$ spectrometer. All spectra were referenced to $\mathrm{Me}_{4} \mathrm{Si}, 85 \%$ $\mathrm{H}_{3} \mathrm{PO}_{4}, \mathrm{Me}_{4} \mathrm{Sn}$ or $\mathrm{K}_{2} \mathrm{PtCl}_{6}$ as appropriate. Carbon-13 NMR data were assigned with recourse to the 2D (HSQC, HMBC) spectra; detailed connectivity and ${ }^{29} \mathrm{Si}$ chemical shifts were assessed using ${ }^{1} \mathrm{H}-\mathrm{X}$ HMBC spectra $\left(\mathrm{X}={ }^{29} \mathrm{Si} ;{ }^{119} \mathrm{Sn} ;{ }^{31} \mathrm{P}\right)$. Elemental analyses were obtained by Mr S. Boyer of the London Metropolitan University Elemental Analysis Service.

\section{Synthesis}

${ }^{n} \mathbf{B u}_{3} \mathbf{S n C} \equiv \mathbf{C C H}_{2} \mathbf{C l}$ (1). In a modification of literature procedure, a solution of propargyl chloride $(2.24 \mathrm{~g}, 3.0 \times$ $10^{-2} \mathrm{~mol}$ ) in THF ( $c a .20 \mathrm{~cm}^{3}$ ) was cooled to $-78{ }^{\circ} \mathrm{C}$ before the drop-wise addition of ${ }^{n} \mathrm{BuLi}\left(2.5 \mathrm{M}, 6.0 \mathrm{~cm}^{3}, 1.5 \times 10^{-2} \mathrm{~mol}\right)$. The mixture was stirred for $30 \mathrm{~min}$., after which time ${ }^{n} \mathrm{Bu}_{3} \mathrm{SnCl}$ $\left(4.40 \mathrm{~cm}^{3}, 1.5 \times 10^{-2} \mathrm{~mol}\right)$ as solution in THF $\left(c a .10 \mathrm{~cm}^{3}\right)$ was added drop-wise, resulting in formation of a yellow solution. 
The mixture was held at $-78^{\circ} \mathrm{C}$ for a further $30 \mathrm{~min}$. with continued stirring before being allowed to warm to ambient temperature overnight. Solvent and excess $\mathrm{HC} \equiv \mathrm{CCH}_{2} \mathrm{Cl}$ were removed under reduced pressure and the product extracted with pentane, stripped of volatiles and dried in vacuo as yellow oil. Yield: $5.09 \mathrm{~g}, 94 \%$. NMR $\left(\mathrm{C}_{6} \mathrm{D}_{6}, 30{ }^{\circ} \mathrm{C}\right):{ }^{1} \mathrm{H}-\mathrm{NMR}: \delta_{\mathrm{H}} 0.91(\mathrm{t}$, $\left.{ }^{3} J_{\mathrm{HH}} 7.3 \mathrm{~Hz}, 9 \mathrm{H}, \mathrm{CH}_{3}\right), 0.97\left(\mathrm{t},{ }^{3} J_{\mathrm{HH}} 6 \mathrm{~Hz}, J_{\mathrm{SnH}} 54 \mathrm{~Hz}, 6 \mathrm{H}\right.$, $\left.\mathrm{CH}_{2} \mathrm{Sn}\right), 1.34$ (m, 6H, $\mathrm{CH}_{3} \mathrm{CH}_{2}$ ), 1.61 (m, 6H, $\left.\mathrm{CH}_{2} \mathrm{CH}_{2} \mathrm{Sn}\right), 3.70$ (s, $\left.J_{\mathrm{SnH}} 9.6 \mathrm{~Hz}, 2 \mathrm{H}, \mathrm{CH}_{2} \mathrm{Cl}\right) .{ }^{13} \mathrm{C}\left\{{ }^{1} \mathrm{H}\right\}-\mathrm{NMR}: \delta_{\mathrm{C}} 11.3\left(\mathrm{~s}, \underline{C}_{2} \mathrm{Sn}\right.$, $\left.{ }^{1} J_{117 \mathrm{SnC}} 365 \mathrm{~Hz},{ }^{1} J_{119 \mathrm{SnC}} 382 \mathrm{~Hz}\right), 13.9\left(\mathrm{~s}, \underline{C} \mathrm{H}_{3}\right), 27.3$ (s, $\left.\underline{\mathrm{CH}_{2}} \mathrm{CH}_{2} \mathrm{Sn},{ }^{2} J_{117 \mathrm{SnC}} 58 \mathrm{~Hz},{ }^{2} J_{119 \mathrm{SnC}} 60 \mathrm{~Hz}\right), 29.3\left(\mathrm{~s}, \mathrm{CH}_{3} \underline{C} \mathrm{H}_{2}\right.$, $\left.{ }^{3} J_{\mathrm{SnC}} 24 \mathrm{~Hz}\right), 31.2\left(\mathrm{~s}, J_{\mathrm{SnC}} 8 \mathrm{~Hz}, \mathrm{CH}_{2} \mathrm{Cl}\right), 91.1$ (s, $\underline{C}=\mathrm{CCH}_{2} \mathrm{Cl}$ ), 105 (s, C $\equiv \underline{C} \mathrm{CH}_{2} \mathrm{Cl}$ ). ${ }^{119} \mathrm{Sn}\left\{{ }^{1} \mathrm{H}\right\}-\mathrm{NMR}: \delta_{\mathrm{Sn}}-65.1$. Anal. Found: C, 49.44; H, 7.86. Calcd for $\mathrm{C}_{15} \mathrm{H}_{29}$ ClSn: C, 49.56; $\mathrm{H}, 8.04$.

$\mathbf{P h}_{3} \mathbf{S n C} \equiv \mathbf{C C H}_{2} \mathbf{C l}$ (2). As for 1, using propargyl chloride $\left(2.03 \mathrm{~g}, 2.7 \times 10^{-2} \mathrm{~mol}\right),{ }^{n} \mathrm{BuLi}\left(2.5 \mathrm{M}, 5.4 \mathrm{~cm}^{3}, 1.3 \times 10^{-2} \mathrm{~mol}\right)$ and $\mathrm{Ph}_{3} \mathrm{SnCl}\left(5.25 \mathrm{~g}, 1.3 \times 10^{-2} \mathrm{~mol}\right)$. Isolated as yellow oil. Yield: $3.96 \mathrm{~g}, 72 \%$. NMR $\left(\mathrm{C}_{6} \mathrm{D}_{6}, 30{ }^{\circ} \mathrm{C}\right):{ }^{1} \mathrm{H}-\mathrm{NMR}: \delta_{\mathrm{H}} 3.67$ (s, $\left.J_{\mathrm{SnH}} 10.5 \mathrm{~Hz}, 2 \mathrm{H}, \mathrm{CH}_{2} \mathrm{Cl}\right), 7.10-7.20\left(\mathrm{~m}, 9 \mathrm{H}, \mathrm{m} / p-\mathrm{C}_{6} H_{5}\right)$, 7.60-7.65 (m, $\left.J_{\mathrm{SnH}} 55 \mathrm{~Hz}, 6 \mathrm{H}, o-\mathrm{C}_{6} H_{5}\right) .{ }^{13} \mathrm{C}\left\{{ }^{1} \mathrm{H}\right\}-\mathrm{NMR}: \delta_{\mathrm{C}} 30.8(\mathrm{~s}$, $\left.J_{\mathrm{SnC}} 10 \mathrm{~Hz}, \mathrm{CH}_{2} \mathrm{Cl}\right), 88.5\left(\mathrm{~s}, \underline{C}=\mathrm{CCH}_{2} \mathrm{Cl}\right), 106.8\left(\mathrm{~s}, \mathrm{C} \equiv \underline{C C H}_{2} \mathrm{Cl}\right)$, 128.8 (s, $\left.p-\underline{C}_{6} \mathrm{H}_{5}\right), 129.5\left(\mathrm{~s}, m-\underline{C}_{6} \mathrm{H}_{5}\right), 130.1\left(\mathrm{~s}, \mathrm{i}-\underline{C}_{6} \mathrm{H}_{5}\right), 136.7$ (s, $\left.{ }^{o-} \underline{C}_{6} \mathrm{H}_{5}\right) \cdot{ }^{119} \mathrm{Sn}\left\{{ }^{1} \mathrm{H}\right\}-\mathrm{NMR}: \delta_{\mathrm{Sn}}-169.5$. Anal. Found: C, 59.63; H, 4.12. Calcd for $\mathrm{C}_{20} \mathrm{H}_{17}$ ClSn: C, 59.55; H, 4.05.

$\mathbf{M e}_{2} \mathbf{P h S i C} \equiv \mathbf{C C H}_{2} \mathbf{C l}$ (3). As for 1, using propargyl chloride $\left(3.73 \mathrm{~g}, 5.0 \times 10^{-2} \mathrm{~mol}\right),{ }^{n} \mathrm{BuLi}\left(2.5 \mathrm{M}, 10.0 \mathrm{~cm}^{3}, 2.5 \times\right.$ $\left.10^{-2} \mathrm{~mol}\right)$ and $\mathrm{Me}_{2} \mathrm{PhSiCl}\left(4.26 \mathrm{~g}, 2.5 \times 10^{-2} \mathrm{~mol}\right)$. The crude product was distilled at $66^{\circ} \mathrm{C}, 8.1 \times 10^{-1}$ mbar to afford colourless oil. Yield: $4.98 \mathrm{~g}, 96 \%$. NMR $\left(\mathrm{C}_{6} \mathrm{D}_{6}, 30{ }^{\circ} \mathrm{C}\right):{ }^{1} \mathrm{H}-\mathrm{NMR}$ : $\delta_{\mathrm{H}} 0.32\left(\mathrm{~s}, J_{\mathrm{SiH}} 8 \mathrm{~Hz}, 6 \mathrm{H}, \mathrm{SiCH}_{3}\right), 3.21\left(\mathrm{~s}, 2 \mathrm{H}, \mathrm{CH}_{2} \mathrm{Cl}\right), 7.14-7.18$ $\left(\mathrm{m}, 3 \mathrm{H}, \mathrm{m} / \mathrm{p}-\mathrm{C}_{6} H_{5}\right), 7.55-7.59\left(\mathrm{~m}, 2 \mathrm{H}, o-\mathrm{C}_{6} H_{5}\right) .{ }^{13} \mathrm{C}\left\{{ }^{1} \mathrm{H}\right\}-\mathrm{NMR}$ : $\delta_{\mathrm{C}}-1.2\left(\mathrm{~s}, \mathrm{SiCH}_{3},{ }^{1} J_{\mathrm{SiC}} 58 \mathrm{~Hz}\right.$ ), $30.5\left(\mathrm{~s}, \mathrm{CH}_{2} \mathrm{Cl}\right), 90.1$ (s, $\underline{C}=\mathrm{CCH}_{2} \mathrm{Cl}$ ), 102.0 (s, C $\left.\equiv \underline{C C H}_{2} \mathrm{Cl}\right) .{ }^{29} \mathrm{Si}\left\{{ }^{1} \mathrm{H}\right\}-\mathrm{NMR}: \delta_{\mathrm{Si}}-21.6$. Anal. Found: C, 63.18; H, 6.14. Calcd for $\mathrm{C}_{11} \mathrm{H}_{13}$ ClSi: C, 63.29; H, 6.28.

${ }^{\mathrm{i}} \mathbf{P r}_{3} \mathbf{S i C} \equiv \mathbf{C C H}_{2} \mathbf{C l}$ (4). As for 1, using propargyl chloride $\left(6.24 \mathrm{~g}, 8.4 \times 10^{-2} \mathrm{~mol}\right),{ }^{n} \mathrm{BuLi}\left(2.5 \mathrm{M}, 16.8 \mathrm{~cm}^{3}, 4.2 \times\right.$ $\left.10^{-2} \mathrm{~mol}\right)$ and ${ }^{\mathrm{i}} \mathrm{Pr}_{3} \mathrm{SiCl}\left(8.06 \mathrm{~g}, 4.2 \times 10^{-2} \mathrm{~mol}\right)$. The crude product was distilled at $52{ }^{\circ} \mathrm{C}, 3.0 \times 10^{-1}$ mbar to afford colourless oil. Yield: $5.76 \mathrm{~g}, 60 \%$. NMR $\left(\mathrm{C}_{6} \mathrm{D}_{6}, 30{ }^{\circ} \mathrm{C}\right)$ : ${ }^{1} \mathrm{H}-\mathrm{NMR}$ : $\delta_{\mathrm{H}} 1.03\left(\mathrm{~m},{ }^{3} \mathrm{H}, \mathrm{SiCH}\right), 1.11\left(\mathrm{~d},{ }^{3} J_{\mathrm{HH}} 6.5 \mathrm{~Hz}, 18 \mathrm{H}, \mathrm{CH}_{3}\right), 3.53(\mathrm{~s}$, $2 \mathrm{H}, \mathrm{CH}_{2} \mathrm{Cl}$ ). ${ }^{13} \mathrm{C}\left\{{ }^{1} \mathrm{H}\right\}-\mathrm{NMR}: \delta_{\mathrm{C}} 11.5\left(\mathrm{~s}, \mathrm{Si} \underline{\mathrm{H}},{ }^{1} J_{\mathrm{SiC}} 57 \mathrm{~Hz}\right), 18.8$ $\left(\mathrm{s}, \underline{\mathrm{C}} \mathrm{H}_{3}\right), 30.6\left(\mathrm{~s}, \mathrm{CH}_{2} \mathrm{Cl}\right), 88.4$ (s, $\left.\underline{C}=\mathrm{CCH}_{2} \mathrm{Cl}\right), 102.7$ (s, $\left.\mathrm{C} \equiv \underline{\bar{C}} \mathrm{CH}_{2} \mathrm{Cl}\right) .{ }^{29} \mathrm{Si}\left\{{ }^{1} \mathrm{H}\right\}-\mathrm{NMR}: \delta_{\mathrm{Si}}-1.68$. Anal. Found: C, 62.38; $\mathrm{H}$, 9.85. Calcd for $\mathrm{C}_{12} \mathrm{H}_{23} \mathrm{ClSi}$ : C, 62.43; $\mathrm{H}, 10.04$.

${ }^{n} \mathbf{P r}_{3} \mathbf{S i C} \equiv \mathbf{C C H}_{2} \mathbf{C l}$ (5). As for $\mathbf{1}$, using propargyl chloride $\left(1.62 \mathrm{~g}, 2.2 \times 10^{-2} \mathrm{~mol}\right),{ }^{n} \operatorname{BuLi}\left(2.5 \mathrm{M}, 4.35 \mathrm{~cm}^{3}, 1.1 \times\right.$ $\left.10^{-2} \mathrm{~mol}\right)$ and ${ }^{n} \mathrm{Pr}_{3} \mathrm{SiCl}\left(2.09 \mathrm{~g}, 1.1 \times 10^{-2} \mathrm{~mol}\right)$. Obtained as orange oil. Yield: $2.33 \mathrm{~g}, 93 \%$. NMR $\left(\mathrm{C}_{6} \mathrm{D}_{6}, 30{ }^{\circ} \mathrm{C}\right):{ }^{1} \mathrm{H}-\mathrm{NMR}$ : $\delta_{\mathrm{H}} 0.60\left(\mathrm{~m}, 6 \mathrm{H}, \mathrm{SiCH}_{2}\right), 0.99\left(\mathrm{t},{ }^{3} J_{\mathrm{HH}} 7.2 \mathrm{~Hz}, 9 \mathrm{H}, \mathrm{CH}_{3}\right), 1.47(\mathrm{~m}$, $6 \mathrm{H}, \mathrm{CH}_{3} \mathrm{CH}_{2}$ ), $3.55\left(\mathrm{~s}, 2 \mathrm{H}, \mathrm{CH}_{2} \mathrm{Cl}\right) .{ }^{13} \mathrm{C}\left\{{ }^{1} \mathrm{H}\right\}-\mathrm{NMR}: \delta_{\mathrm{C}} 16.2(\mathrm{~s}$, $\underline{C} \mathrm{H}_{2} \mathrm{Si},{ }^{1} J_{\mathrm{SiC}} 56 \mathrm{~Hz}$ ), $17.9\left(\mathrm{~s}, \underline{C} \mathrm{H}_{3}\right), 18.4\left(\mathrm{~s}, \underline{C} \mathrm{H}_{2} \mathrm{CH}_{2} \mathrm{Si},{ }^{2} J_{\mathrm{SiC}}\right.$ $6 \mathrm{~Hz}), 30.7\left(\mathrm{~s}, \mathrm{CH}_{2} \mathrm{Cl}\right), 90.2\left(\mathrm{~s}, \underline{C}=\mathrm{CCH}_{2} \mathrm{Cl}\right), 101.8$ (s, $\left.\mathrm{C} \equiv \underline{C} \mathrm{CH}_{2} \mathrm{Cl}\right) .{ }^{29} \mathrm{Si}\left\{{ }^{1} \mathrm{H}\right\}-\mathrm{NMR}: \delta_{\mathrm{Si}}-13.0$. Anal. Found: C, 62.87; $\mathrm{H}$, 9.79. Calcd for $\mathrm{C}_{12} \mathrm{H}_{23} \mathrm{ClSi}$ : C, 62.43; $\mathrm{H}, 10.04$.

${ }^{n} \mathrm{Bu}_{3} \mathrm{SiC} \equiv \mathrm{CCH}_{2} \mathrm{Cl}$ (6). As for 1, using propargyl chloride (1.92 g, $\left.2.5 \times 10^{-2} \mathrm{~mol}\right),{ }^{n} \mathrm{BuLi}\left(2.5 \mathrm{M}, 5.2 \mathrm{~cm}^{3}, 1.3 \times 10^{-2} \mathrm{~mol}\right)$ and ${ }^{n} \mathrm{Bu}_{3} \mathrm{SiCl}\left(3.02 \mathrm{~g}, 1.29 \times 10^{-2} \mathrm{~mol}\right)$. Obtained as orange oil. Yield: $3.08 \mathrm{~g}, 88 \%$. NMR $\left(\mathrm{C}_{6} \mathrm{D}_{6}, 30{ }^{\circ} \mathrm{C}\right):{ }^{1} \mathrm{H}-\mathrm{NMR}: \delta_{\mathrm{H}} 0.67(\mathrm{~m}$, $\left.6 \mathrm{H}, \mathrm{SiCH}_{2}\right), 0.92\left(\mathrm{t},{ }^{3} \mathrm{~J}_{\mathrm{HH}} 7.3 \mathrm{~Hz}, 9 \mathrm{H}, \mathrm{CH}_{3}\right), 1.38(\mathrm{~m}, 6 \mathrm{H}$, $\mathrm{CH}_{2} \mathrm{CH}_{2} \mathrm{Si}$ ), $1.46\left(\mathrm{~m}, 6 \mathrm{H}, \mathrm{CH}_{3} \mathrm{CH}_{2} \mathrm{CH}_{2}\right), 3.56$ (s, 2H, $\left.\mathrm{CH}_{2} \mathrm{Cl}\right)$. ${ }^{13} \mathrm{C}\left\{{ }^{1} \mathrm{H}\right\}$-NMR: $\delta_{\mathrm{C}} 13.3\left(\mathrm{~s}, \underline{C} \mathrm{H}_{2} \mathrm{Si},{ }^{1} J_{\mathrm{SiC}} 57 \mathrm{~Hz}\right), 14.0\left(\mathrm{~s}, \underline{C} \mathrm{H}_{3}\right), 26.5$ (s, $\mathrm{CH}_{3} \underline{\mathrm{CH}_{2}} \mathrm{CH}_{2}$ ), 26.8 (s, $\underline{\mathrm{C}} \mathrm{H}_{2} \mathrm{CH}_{2} \mathrm{Si}, J_{\mathrm{SiC}} 6 \mathrm{~Hz}$ ), 30.7 (s, $\mathrm{CH}_{2} \mathrm{Cl}$ ), 90.3 (s, $\left.\underline{C}=\mathrm{CCH}_{2} \mathrm{Cl}\right), 101.8$ (s, C $\left.\equiv \underline{C} \mathrm{CH}_{2} \mathrm{Cl}\right) .{ }^{29} \mathrm{Sn}\left\{{ }^{1} \mathrm{H}\right\}-\mathrm{NMR}$ : $\delta_{\mathrm{Si}}$-11.3. Anal. Found: $\mathrm{C}, 66.39 ; \mathrm{H}, 10.02$. Calcd for $\mathrm{C}_{15} \mathrm{H}_{29}$ ClSi: C, 66.01; H, 10.71.

$\mathbf{P h}_{3} \mathbf{S i C} \equiv \mathbf{C C H}_{2} \mathbf{C l}$ (7). As for 1, using propargyl chloride $\left(1.00 \mathrm{~g}, 1.03 \times 10^{-2} \mathrm{~mol}\right),{ }^{n} \mathrm{BuLi}\left(2.5 \mathrm{M}, 2.7 \mathrm{~cm}^{3}, 6.7 \times\right.$ $\left.10^{-3} \mathrm{~mol}\right)$ and ${ }^{n} \mathrm{Bu}_{3} \mathrm{SiCl}\left(3.83 \mathrm{~g}, 1.3 \times 10^{-3} \mathrm{~mol}\right)$. The crude product was sublimed under reduced pressure $(23.0 \times$ $10^{-3} \mathrm{mbar}$ ) to afford a colourless solid. Yield: $3.04 \mathrm{~g}, 89 \%$. NMR $\left(\mathrm{C}_{6} \mathrm{D}_{6}, 30{ }^{\circ} \mathrm{C}\right):{ }^{1} \mathrm{H}-\mathrm{NMR}: \delta_{\mathrm{H}} 3.49\left(\mathrm{~s}, 2 \mathrm{H}, \mathrm{CH}_{2} \mathrm{Cl}\right), 7.14-7.16$ $\left(\mathrm{m}, 9 \mathrm{H}, m / p-\mathrm{C}_{6} H_{5}\right), 7.73-7.78\left(\mathrm{~m}, 6 \mathrm{H}, o-\mathrm{C}_{6} H_{5}\right) .{ }^{13} \mathrm{C}\left\{{ }^{1} \mathrm{H}\right\}-\mathrm{NMR}$ : $\delta_{\mathrm{C}} 30.4\left(\mathrm{~s}, \mathrm{CH}_{2} \mathrm{Cl}\right), 87.6\left(\mathrm{~s}, \underline{C}=\mathrm{CCH}_{2} \mathrm{Cl}\right), 104.9\left(\mathrm{~s}, \mathrm{C} \equiv \underline{C_{C H}} \mathrm{Cl}\right)$, $128.4\left(\mathrm{~s}, p-\underline{C}_{6} \mathrm{H}_{5}\right), 130.4\left(\mathrm{~s}, m-\underline{C}_{6} \mathrm{H}_{5}\right), 133.4\left(\mathrm{~s}, \mathrm{i}-\underline{C}_{6} \overline{\mathrm{H}}_{5}\right), 136.0$ (s, o- $\left.\underline{C}_{6} \mathrm{H}_{5}\right) .{ }^{29} \mathrm{Sn}\left\{{ }^{1} \mathrm{H}\right\}-\mathrm{NMR}: \delta_{\mathrm{Si}}-28.8$. Anal. Found: C, 75.68; $\mathrm{H}$, 5.11. Calcd for $\mathrm{C}_{21} \mathrm{H}_{17}$ ClSi: C, 75.77; H, 5.15.

${ }^{n} \mathbf{B u}_{3} \mathbf{S n C} \equiv \mathbf{C C H}_{2} \mathbf{P P h}_{2} \quad$ (8). To an ethereal solution (ca. $\left.20 \mathrm{~cm}^{3}\right)$ of $\mathrm{HPPh}_{2}\left(0.375 \mathrm{~g}, 2.02 \times 10^{-3} \mathrm{~mol}\right)$ held at $-78{ }^{\circ} \mathrm{C}$ was added drop-wise ${ }^{n} \mathrm{BuLi}\left(2.5 \mathrm{M}, 0.808 \mathrm{~cm}^{3}, 2.02 \times\right.$ $\left.10^{-3} \mathrm{~mol}\right)$; the mixture was stirred for $30 \mathrm{~min}$. A solution of 1 $\left(0.733 \mathrm{~g}, 2.02 \times 10^{-3} \mathrm{~mol}\right)$ in ether $\left(c a .10 \mathrm{~cm}^{3}\right)$ was then added drop-wise and the mixture maintained at $-78^{\circ} \mathrm{C}$ while stirring for $30 \mathrm{~min}$. The mixture was allowed to warm to ambient temperature while stirring overnight. Volatiles were removed under reduced pressure and the product extracted with pentane; the solvent was removed and the product dried in vacuo to afford yellow oil. Yield: $0.800 \mathrm{~g}\left(4: 1 \mathrm{8}: \mathrm{SnBu}_{4}\right)$. NMR $\left(\mathrm{C}_{6} \mathrm{D}_{6}, 30{ }^{\circ} \mathrm{C}\right)$ : ${ }^{1} \mathrm{H}-\mathrm{NMR}: \delta_{\mathrm{H}} 0.93\left(\mathrm{~m}, \mathrm{CH}_{3}\right), 1.36\left(\mathrm{~m}, 12 \mathrm{H}, 2 \times \mathrm{CH}_{2}\right), 1.58(\mathrm{~m}$, $6 \mathrm{H}, \mathrm{CH}_{2}$ ), 2.87 (d, $J_{\mathrm{PH}} 1.6 \mathrm{~Hz}, J_{117 \mathrm{SnC}} 8.7 \mathrm{~Hz}, J_{119 \mathrm{SnC}} 12.4 \mathrm{~Hz}$, $\left.2 \mathrm{H}, \mathrm{CH}_{2} \mathrm{P}\right), 7.02-7.13\left(\mathrm{~m}, 6 \mathrm{H}, \mathrm{m} / \mathrm{p}-\mathrm{C}_{6} \mathrm{H}_{5}\right), 7.43-7.51(\mathrm{~m}, 4 \mathrm{H}$, $\left.o-\mathrm{C}_{6} H_{5}\right) .{ }^{13} \mathrm{C}\left\{{ }^{1} \mathrm{H}\right\}-\mathrm{NMR}: \delta_{\mathrm{C}} 11.3\left(\mathrm{~s}, \underline{C} \mathrm{H}_{2} \mathrm{Sn},{ }^{1} J_{117 \mathrm{SnC}} 366 \mathrm{~Hz}\right.$, $\left.{ }^{1} J_{119 \mathrm{SnC}} 383 \mathrm{~Hz}\right), 13.9\left(\mathrm{~s}, \underline{\mathrm{C}} \mathrm{H}_{3}\right), 20.4\left(\mathrm{~d},{ }^{1} J_{\mathrm{PC}} 18 \mathrm{~Hz}, \underline{\mathrm{CH}_{2}} \mathrm{PPh}_{2}\right)$, $27.4\left(\mathrm{~s}, \quad \underline{\mathrm{C}} \mathrm{H}_{2} \mathrm{CH}_{2} \mathrm{Sn}, J_{\mathrm{SnC}} 58 \mathrm{~Hz}\right) 85.0$ (d, $J_{\mathrm{PC}} 6 \mathrm{~Hz}$, $\left.\underline{C}=\mathrm{CCH}_{2} \mathrm{PPh}_{2}\right), 106.8\left(\mathrm{~d}, J_{\mathrm{PC}} 5 \mathrm{~Hz}, \mathrm{C} \equiv{ }_{C} \mathrm{CH}_{2} \mathrm{PPh}_{2}\right), 128.6\left(\mathrm{~d}, J_{\mathrm{PC}}\right.$ $6 \mathrm{~Hz}, m-C_{6} \mathrm{H}_{5}$ ), $128.9\left(\mathrm{~s}, p-C_{6} \mathrm{H}_{5}\right), 133.2\left(\mathrm{~d}, J_{\mathrm{PC}} 19 \mathrm{~Hz}, o-C_{6} \mathrm{H}_{5}\right.$ ), $138.8\left(\mathrm{~d}, J_{\mathrm{PC}} 17 \mathrm{~Hz}, \mathrm{i}-C_{6} \mathrm{H}_{5}\right) .{ }^{31} \mathrm{P}\left\{{ }^{1} \mathrm{H}\right\}-\mathrm{NMR}: \delta_{\mathrm{P}}-13.4\left(\mathrm{~s}, J_{\mathrm{SnP}} 14.5\right.$ Hz). ${ }^{119} \mathrm{Sn}\left\{{ }^{1} \mathrm{H}\right\}-\mathrm{NMR}: \delta_{\mathrm{Sn}}-68.5\left(\mathrm{~d}, J_{\mathrm{SnP}} 14.5 \mathrm{~Hz}, 4 \mathrm{Sn}\right),-12.0$ (s, $\left.1 \mathrm{Sn}, \mathrm{Bu}_{4} \mathrm{Sn}\right)$.

$\mathbf{P h}_{3} \mathbf{S n C} \equiv \mathbf{C C H}_{2} \mathbf{P P h}$ (9). As for 8, using $\mathrm{HPPh}_{2}$ (0.309 g, $\left.1.66 \times 10^{-3} \mathrm{~mol}\right),{ }^{n} \mathrm{BuLi}\left(2.1 \mathrm{M}, 0.80 \mathrm{~cm}^{3}, 1.66 \times 10^{-3} \mathrm{~mol}\right)$ and $2\left(0.876 \mathrm{~g}, 1.66 \times 10^{-3} \mathrm{~mol}\right)$. Isolated as yellow oil. $\mathrm{NMR}\left(\mathrm{C}_{6} \mathrm{D}_{6}\right.$, $\left.30{ }^{\circ} \mathrm{C}\right):{ }^{1} \mathrm{H}-\mathrm{NMR}: \delta_{\mathrm{H}} 2.84\left(\mathrm{~d}, J_{\mathrm{PH}} 3.0 \mathrm{~Hz}, J_{\mathrm{SnH}} 14.8 \mathrm{~Hz}, 2 \mathrm{H}\right.$, $\left.\mathrm{CH}_{2} \mathrm{P}\right)$, 6.89-7.20 (m, Ar, $\left.m / p-\mathrm{C}_{6} H_{5}\right), 7.37-7.70(\mathrm{~m}, A r, 6 \mathrm{H}$, $o$ - $\left.{ }_{6} H_{5}\right) .{ }^{13} \mathrm{C}\left\{{ }^{1} \mathrm{H}\right\}-\mathrm{NMR}: \delta_{\mathrm{C}} 20.2\left(\mathrm{~d}, J_{\mathrm{PC}} 21 \mathrm{~Hz}, J_{\mathrm{SnC}} 11.9 \mathrm{~Hz}\right.$, $\mathrm{CH}_{2} \mathrm{P}$ ), 82.8 (d, $\left.J_{\mathrm{PC}} 6 \mathrm{~Hz}, J_{\mathrm{SnC}} 3.4 \mathrm{~Hz}, \mathrm{C}=\underline{C} \mathrm{CH}_{2} \mathrm{P}\right), 109.3\left(\mathrm{~d}, J_{\mathrm{PC}}\right.$ $\left.3.4 \mathrm{~Hz}, \underline{C} \equiv \mathrm{CCH}_{2} \mathrm{P}\right) .{ }^{31} \mathrm{P}\left\{{ }^{1} \mathrm{H}\right\}-\mathrm{NMR}: \delta_{\mathrm{P}}-13.2(\mathrm{~s}) .{ }^{119} \mathrm{Sn}\left\{{ }^{1} \mathrm{H}\right\}-\mathrm{NMR}$ : $\delta_{\text {Sn }}-168.4\left(J_{\text {SnP }} 14.8 \mathrm{~Hz}, 1 \mathrm{Sn}\right),-98.3$ (s, BuSnPh$\left.{ }_{3}, 1 \mathrm{Sn}\right)$.

$\mathbf{M e}_{2} \mathbf{P h S i C} \equiv \mathbf{C C H}_{2} \mathbf{P P h}_{2}$ (10). As for 8, using $\mathrm{HPPh}_{2}(0.780 \mathrm{~g}$, $\left.4.24 \times 10^{-3} \mathrm{~mol}\right),{ }^{n} \mathrm{BuLi}\left(2.5 \mathrm{M}, 1.7 \mathrm{~cm}^{3}, 4.24 \times 10^{-3} \mathrm{~mol}\right)$ and $3\left(0.884 \mathrm{~g}, 4.24 \times 10^{-3} \mathrm{~mol}\right)$. Isolated as brown oil. Yield: $1.19 \mathrm{~g}, 78 \%$. NMR $\left(\mathrm{C}_{6} \mathrm{D}_{6}, 30{ }^{\circ} \mathrm{C}\right):{ }^{1} \mathrm{H}-\mathrm{NMR}: \delta_{\mathrm{H}} 0.30(\mathrm{~s}, 6 \mathrm{H}$, $\mathrm{SiCH}_{3}$ ), 2.75 (d, $\left.J_{\mathrm{PH}} 2.9 \mathrm{~Hz}, 2 \mathrm{H}, \mathrm{CH}_{2} \mathrm{P}\right), 7.01-7.09(\mathrm{~m}, 6 \mathrm{H}$, 
$\left.m / p-\mathrm{P}\left(\mathrm{C}_{6} H_{5}\right)_{2}\right), 7.17-7.22\left(\mathrm{~m}, 4 \mathrm{H}, o-\mathrm{P}\left(\mathrm{C}_{6} H_{5}\right)_{2}\right), 7.39-7.46(\mathrm{~m}, 3 \mathrm{H}$, $\left.m / p-\mathrm{SiC}_{6} H_{5}\right), \quad 7.52-7.58 \quad\left(\mathrm{~m}, \quad 2 \mathrm{H}, \quad o-\mathrm{SiC}_{6} H_{5}\right) .{ }^{13} \mathrm{C}\left\{{ }^{1} \mathrm{H}\right\}-\mathrm{NMR}$ : $\delta_{\mathrm{C}}-0.6\left(\mathrm{~s}, \mathrm{Si}_{\underline{C}} \mathrm{H}_{3}\right), 19.9\left(\mathrm{~d}, J_{\mathrm{PC}} 21 \mathrm{~Hz}, \underline{C} \underline{H}_{2} \mathrm{P}\right), 85.7\left(\mathrm{~d}, J_{\mathrm{PC}} 5 \mathrm{~Hz}\right.$, $\left.\underline{C} \equiv \mathrm{CCH}_{2} \mathrm{P}\right), 104.9\left(\mathrm{~d}, J_{\mathrm{PC}} 4 \mathrm{~Hz}, \mathrm{C} \equiv \underline{C} \mathrm{CH}_{2} \mathrm{P}\right), 128.7\left(\mathrm{~d}, J_{\mathrm{PC}} 6.5\right.$ $\left.\mathrm{Hz}, m-C_{6} \mathrm{H}_{5}\right), 129.0\left(\mathrm{~s}, p-C_{6} \mathrm{H}_{5}\right), 129.5\left(\mathrm{~s}, p-C_{6} \mathrm{H}_{5}\right), 133.2$ (d, $\left.J_{\mathrm{PC}} 19.5 \mathrm{~Hz}, o-C_{6} \mathrm{H}_{5}\right), 134.2\left(\mathrm{~s}, o-C_{6} \mathrm{H}_{5}\right), 137.7\left(\mathrm{~s}, \mathrm{i}-C_{6} \mathrm{H}_{5}\right)$, $138.1\left(\mathrm{~d}, J_{\mathrm{PC}} 16 \mathrm{~Hz}, \quad \mathrm{i}-C_{6} \mathrm{H}_{5}\right) .{ }^{31} \mathrm{P}\left\{{ }^{1} \mathrm{H}\right\}-\mathrm{NMR}: \delta_{\mathrm{P}}-13.5$ (s). ${ }^{29} \mathrm{Si}\left\{{ }^{1} \mathrm{H}\right\}$-NMR: $\delta_{\mathrm{Si}}-22.9$. Anal. Found: $\mathrm{C}, 76.89 ; \mathrm{H}, 6.34$. Calcd for $\mathrm{C}_{23} \mathrm{H}_{23}$ PSi: C, 77.06; $\mathrm{H}, 6.47$.

${ }^{\mathrm{i}} \mathbf{P r}_{3} \mathbf{S i C} \equiv \mathbf{C C H}_{2} \mathbf{P P h}_{2}$ (11). As for 8, using $\mathrm{HPPh}_{2}(0.780 \mathrm{~g}$, $\left.4.24 \times 10^{-3} \mathrm{~mol}\right),{ }^{n} \operatorname{BuLi}\left(2.5 \mathrm{M}, 1.7 \mathrm{~cm}^{3}, 4.24 \times 10^{-3} \mathrm{~mol}\right)$ and 4 $\left(0.976 \mathrm{~g}, 4.24 \times 10^{-3} \mathrm{~mol}\right)$. Isolated as orange oil. Yield: $1.45 \mathrm{~g}$, 90\%. NMR $\left(\mathrm{C}_{6} \mathrm{D}_{6}, 30{ }^{\circ} \mathrm{C}\right):{ }^{1} \mathrm{H}-\mathrm{NMR}: \delta_{\mathrm{H}} 1.01(\mathrm{~m}, 3 \mathrm{H}, \mathrm{SiCH}), 1.09$ (d, $\left.{ }^{3} J_{\mathrm{HH}} 6.8 \mathrm{~Hz}, 18 \mathrm{H}, \mathrm{CH}_{3}\right), 2.75\left(\mathrm{~d}, J_{\mathrm{PH}} 2.3 \mathrm{~Hz}, 2 \mathrm{H}, \mathrm{CH}_{2} \mathrm{P}\right)$, 7.02-7.12 (m, 6H, m/p-P( $\left.\left.\mathrm{C}_{6} H_{5}\right)_{2}\right), 7.39-7.47\left(\mathrm{~m}, 4 \mathrm{H}, o-\mathrm{P}\left(\mathrm{C}_{6} H_{5}\right)_{2}\right)$. ${ }^{13} \mathrm{C}\left\{{ }^{1} \mathrm{H}\right\}$-NMR: $\delta_{\mathrm{C}} 11.7\left(\mathrm{~s}, \mathrm{Si} \underline{\mathrm{CH}},{ }^{1} J_{\mathrm{SiC}} 56 \mathrm{~Hz}\right), 18.9\left(\mathrm{~s}, J_{\mathrm{SiC}} 16 \mathrm{~Hz}\right.$, $\left.\underline{C}_{3}\right), 19.9\left(\mathrm{~d}, J_{\mathrm{PC}} 19.5 \mathrm{~Hz}, \underline{\underline{C}} \mathrm{H}_{2} \mathrm{P}\right), 83.3\left(\mathrm{~d}, J_{\mathrm{PC}} 5 \mathrm{~Hz}, \underline{C} \equiv \mathrm{CCH}_{2} \mathrm{P}\right.$ ), $104.7\left(\mathrm{~d}, J_{\mathrm{PC}} 4 \mathrm{~Hz}, \mathrm{C} \equiv \underline{C}^{-} \overline{\mathrm{H}}_{2} \mathrm{P}\right), 128.7\left(\mathrm{~d}, J_{\mathrm{PC}} 6.5 \mathrm{~Hz}, m-C_{6} \mathrm{H}_{5}\right)$, 129.0 (s, $p-C_{6} \mathrm{H}_{5}$ ), 133.1 (d, $\left.J_{\mathrm{PC}} 19 \mathrm{~Hz}, o-C_{6} \mathrm{H}_{5}\right), 138.3$ (d, $J_{\mathrm{PC}} 16$ $\left.\mathrm{Hz}, \mathrm{i}-\mathrm{C}_{6} \mathrm{H}_{5}\right) .{ }^{31} \mathrm{P}\left\{{ }^{1} \mathrm{H}\right\}-\mathrm{NMR}: \delta_{\mathrm{P}}-13.5$ (s, $\left.J_{\mathrm{SiP}} 20 \mathrm{~Hz}\right) .{ }^{29} \mathrm{Si}\left\{{ }^{1} \mathrm{H}\right\}-$ NMR: $\delta_{\mathrm{Si}}-3.03$. Anal. Found: C, 75.77; $\mathrm{H}, 8.64$. Calcd for $\mathrm{C}_{24} \mathrm{H}_{33}$ PSi: C, 75.74; H, 8.74.

${ }^{n} \mathbf{P r}_{3} \mathbf{S i C} \equiv \mathbf{C C H}_{2} \mathbf{P P h}_{2}$ (12). As for 8, using $\mathrm{HPPh}_{2}(0.650 \mathrm{~g}$, $\left.3.49 \times 10^{-3} \mathrm{~mol}\right),{ }^{n} \mathrm{BuLi}\left(2.5 \mathrm{M}, 1.4 \mathrm{~cm}^{3}, 3.49 \times 10^{-3} \mathrm{~mol}\right)$ and 5 $\left(0.805 \mathrm{~g}, 3.49 \times 10^{-3} \mathrm{~mol}\right)$. Isolated as brown oil. Yield: $1.00 \mathrm{~g}$, 80\%. NMR $\left(\mathrm{C}_{6} \mathrm{D}_{6}, 30{ }^{\circ} \mathrm{C}\right):{ }^{1} \mathrm{H}-\mathrm{NMR}: \delta_{\mathrm{H}} 0.58\left(\mathrm{~m}, 6 \mathrm{H}, \mathrm{SiCH}_{2}\right)$, $0.99\left(\mathrm{t},{ }^{3} J_{\mathrm{HH}} 7.0 \mathrm{~Hz}, 9 \mathrm{H}, \mathrm{CH}_{3}\right), 1.42\left(\mathrm{~m}, 6 \mathrm{H}, \mathrm{CH}_{2} \mathrm{CH}_{2} \mathrm{Si}\right), 2.75$ (d, $\left.J_{\mathrm{PH}} 2.5 \mathrm{~Hz}, 2 \mathrm{H}, \mathrm{CH}_{2} \mathrm{P}\right), 7.04-7.12\left(\mathrm{~m}, 6 \mathrm{H}, m / p-\mathrm{P}\left(\mathrm{C}_{6} H_{5}\right)_{2}\right)$, 7.40-7.46 (m, 4H, o-P $\left.\left(\mathrm{C}_{6} H_{5}\right)_{2}\right) .{ }^{13} \mathrm{C}\left\{{ }^{1} \mathrm{H}\right\}$-NMR: $\delta_{\mathrm{C}} 16.7\left(\mathrm{~s}, \underline{\mathrm{C}} \mathrm{H}_{2} \mathrm{Si}\right.$, ${ }^{1} J_{\mathrm{SiC}} 56 \mathrm{~Hz}$ ), $18.0\left(\mathrm{~s}, \underline{\mathrm{C}} \mathrm{H}_{3}\right), 18.5\left(\mathrm{~s}, \underline{\mathrm{C}} \mathrm{H}_{2} \mathrm{CH}_{2} \mathrm{Si},{ }^{2} J_{\mathrm{SiC}} 8 \mathrm{~Hz}\right), 19.9$ $\left(\mathrm{d}, J_{\mathrm{PC}} 20 \mathrm{~Hz}, \underline{C} \mathrm{H}_{2} \mathrm{P}\right), 85.4\left(\mathrm{~d}, J_{\mathrm{PC}} 5.4 \mathrm{~Hz}, \underline{C}=\mathrm{CCH}_{2} \mathrm{P}\right), 104.7$ (d, $\left.J_{\mathrm{PC}} 4 \mathrm{~Hz}, \mathrm{C} \equiv \underline{C} \mathrm{CH}_{2} \mathrm{P}\right), 128.5\left(\mathrm{~d}, J_{\mathrm{PC}} 6.6 \mathrm{~Hz}, m-C_{6} \mathrm{H}_{5}\right), 129.0(\mathrm{~s}$, $\left.p-C_{6} \mathrm{H}_{5}\right), 133.2\left(\mathrm{~d}, J_{\mathrm{PC}} 19 \mathrm{~Hz}, o-C_{6} \mathrm{H}_{5}\right), 138.3\left(\mathrm{~d}, J_{\mathrm{PC}} 16.5 \mathrm{~Hz}\right.$, i- $\left.C_{6} \mathrm{H}_{5}\right) .{ }^{31} \mathrm{P}\left\{{ }^{1} \mathrm{H}\right\}$-NMR: $\delta_{\mathrm{P}}-13.6$ (s, $\left.J_{\text {Sip }} 19.7 \mathrm{~Hz}\right) .{ }^{29} \mathrm{Si}\left\{{ }^{1} \mathrm{H}\right\}$-NMR: $\delta_{\mathrm{Si}}-14.8$. Anal. Found: $\mathrm{C}, 75.77 ; \mathrm{H}, 8.59$. Calcd for $\mathrm{C}_{24} \mathrm{H}_{33} \mathrm{PSi}$ : C, $75.74 ; \mathrm{H}, 8.74$.

${ }^{n} \mathbf{B u}_{3} \mathbf{S i C} \equiv \mathbf{C C H}_{2} \mathbf{P P h}_{2}$ (13). As for 8, using $\mathrm{HPPh}_{2}$ (0.650 g, $\left.4.24 \times 10^{-3} \mathrm{~mol}\right),{ }^{n} \operatorname{BuLi}\left(2.5 \mathrm{M}, 1.15 \mathrm{~cm}^{3}, 2.87 \times 10^{-3} \mathrm{~mol}\right)$ and $6\left(0.784 \mathrm{~g}, 1.87 \times 10^{-3} \mathrm{~mol}\right)$. Isolated as brown oil. Yield: $0.95 \mathrm{~g}, 79 \%$. NMR $\left(\mathrm{C}_{6} \mathrm{D}_{6}, 30{ }^{\circ} \mathrm{C}\right):{ }^{1} \mathrm{H}-\mathrm{NMR}: \delta_{\mathrm{H}} 0.6 .3(\mathrm{~m}, 6 \mathrm{H}$, $\left.\mathrm{SiCH}_{2}\right), 0.93\left(\mathrm{t},{ }^{3} J_{\mathrm{HH}} 7.2 \mathrm{~Hz}, 9 \mathrm{H}, \mathrm{CH}_{3}\right), 1.37\left(\mathrm{~m}, 6 \mathrm{H}, \mathrm{CH}_{2} \mathrm{CH}_{2} \mathrm{Si}\right)$, $1.41\left(\mathrm{~m}, 6 \mathrm{H}, \mathrm{CH}_{3} \mathrm{CH}_{2} \mathrm{CH}_{2}\right), 2.76\left(\mathrm{~d}, J_{\mathrm{PH}} 2.3 \mathrm{~Hz}, 2 \mathrm{H}, \mathrm{CH}_{2} \mathrm{P}\right)$, 7.04-7.13 (m, 6H, $\left.m / p-\mathrm{P}\left(\mathrm{C}_{6} H_{5}\right)_{2}\right), 7.41-7.46\left(\mathrm{~m}, 4 \mathrm{H}, o-\mathrm{P}\left(\mathrm{C}_{6} H_{5}\right)_{2}\right)$. ${ }^{13} \mathrm{C}\left\{{ }^{1} \mathrm{H}\right\}-\mathrm{NMR}: \delta_{\mathrm{C}} 13.7\left(\mathrm{~s}, \underline{\mathrm{C}} \mathrm{H}_{2} \mathrm{Si}\right), 14.1\left(\mathrm{~s}, \underline{\mathrm{C}} \mathrm{H}_{3}\right), 19.9$ (d, $J_{\mathrm{PC}}$ $\left.20 \mathrm{~Hz}, \underline{\mathrm{C}} \mathrm{H}_{2} \mathrm{P}\right), 26.7\left(\mathrm{~s}, \mathrm{CH}_{3} \underline{\underline{C}} \mathrm{H}_{2} \mathrm{CH}_{2}\right), 26.9\left(\mathrm{~s}, \underline{\mathrm{C}} \mathrm{H}_{2} \mathrm{CH}_{2} \mathrm{Si}\right), 85.5$ (d, $\left.J_{\mathrm{PC}} 4.8 \mathrm{~Hz}, \underline{C}=\mathrm{CCH}_{2} \mathrm{P}\right), 104.0\left(\mathrm{~d}, J_{\mathrm{PC}} 4.2 \mathrm{~Hz}, \mathrm{C} \equiv \underline{C} \mathrm{CH}_{2} \mathrm{P}\right)$, $128.6\left(\mathrm{~d}, J_{\mathrm{PC}} 6.4 \mathrm{~Hz}, m-C_{6} \mathrm{H}_{5}\right), 129.0\left(\mathrm{~s}, p-C_{6} \mathrm{H}_{5}\right), 133.2\left(\mathrm{~d}, J_{\mathrm{PC}}\right.$ $\left.19 \mathrm{~Hz}, o-C_{6} \mathrm{H}_{5}\right), 138.3\left(\mathrm{~d}, J_{\mathrm{PC}} 15.5 \mathrm{~Hz}, \mathrm{i}-C_{6} \mathrm{H}_{5}\right) .{ }^{31} \mathrm{P}\left\{{ }^{1} \mathrm{H}\right\}-\mathrm{NMR}$ : $\delta_{\mathrm{P}}-13.5\left(\mathrm{~s}, J_{\mathrm{SiP}} 18.0 \mathrm{~Hz}\right) .{ }^{29} \mathrm{Si}\left\{{ }^{1} \mathrm{H}\right\}-\mathrm{NMR}: \delta_{\mathrm{Si}}-12.9$. Anal. Found: C, 76.78; H, 9.32. Calcd for $\mathrm{C}_{27} \mathrm{H}_{39} \mathrm{PSi}$ C, 76.73; H, 9.30.

$\mathbf{M e}_{2} \mathbf{P h S i C} \equiv \mathbf{C C H}_{2} \mathbf{P}\left(\mathrm{SiMe}_{3}\right)_{2}$ (14). In a manner similar to that described for 8, using $\mathrm{HP}\left(\mathrm{SiMe}_{3}\right)_{2}(1.04 \mathrm{~g}, 5.84 \times$ $\left.10^{-3} \mathrm{~mol}\right),{ }^{n} \operatorname{BuLi}\left(2.5 \mathrm{M}, 2.3 \mathrm{~cm}^{3}, 5.75 \times 10^{-3} \mathrm{~mol}\right)$ and 3 $\left(1.25 \mathrm{~g}, 6.00 \times 10^{-3} \mathrm{~mol}\right)$. Isolated as orange oil. Yield: $1.84 \mathrm{~g}$, $90 \%$. NMR $\left(\mathrm{C}_{6} \mathrm{D}_{6}, 30{ }^{\circ} \mathrm{C}\right):{ }^{1} \mathrm{H}-\mathrm{NMR}: \delta_{\mathrm{H}} 0.25\left(\mathrm{~d}, J_{\mathrm{PH}} 4.8 \mathrm{~Hz}, 18 \mathrm{H}\right.$, $\left.2 \times \mathrm{Si}\left(\mathrm{CH}_{3}\right)_{3}\right), 0.44\left(\mathrm{~s}, 2 \times \mathrm{SiCH}_{3}\right), 2.43\left(\mathrm{~d}, J_{\mathrm{PH}} 1.3 \mathrm{~Hz}, 2 \mathrm{H}\right.$,
$\left.\mathrm{CH}_{2} \mathrm{P}\right), 7.20-7.25\left(\mathrm{~m}, 3 \mathrm{H}, \mathrm{m} / \mathrm{p}-\mathrm{SiC}_{6} H_{5}\right), 7.70-7.74(\mathrm{~m}, 2 \mathrm{H}$, $o$-SiC $\left.{ }_{6} H_{5}\right) \cdot{ }^{13} \mathrm{C}\left\{{ }^{1} \mathrm{H}\right\}$-NMR: $\delta_{\mathrm{C}}-0.6\left(\mathrm{~s}, \mathrm{Si}_{\underline{C}} \mathrm{H}_{3}\right), 1.1\left(\mathrm{~d} J_{\mathrm{PC}} 12.5 \mathrm{~Hz}\right.$, $\left.\left.\mathrm{P}\{\mathrm{Si} \underline{C} \mathrm{H})_{3}\right\}_{2}\right), 5.5\left(\mathrm{~d}, J_{\mathrm{PC}} 23 \mathrm{~Hz}, \underline{C} \mathrm{H}_{2} \mathrm{P}\right), 83.3\left(\mathrm{~d}, J_{\mathrm{PC}} 4 \mathrm{~Hz}\right.$, $\left.\underline{C}=\mathrm{CCH}_{2} \mathrm{P}\right), 109.3$ (br., $\left.\mathrm{C} \equiv \underline{C_{C}} \mathrm{CH}_{2} \mathrm{P}\right), 128.2\left(\mathrm{~s}, m-C_{6} \mathrm{H}_{5}\right), 129.6$ (s, $\left.p-C_{6} \mathrm{H}_{5}\right), 134.2\left(\mathrm{~s}, o-C_{6} \mathrm{H}_{5}\right), 137.7\left(\mathrm{~s}, \mathrm{i}-C_{6} \mathrm{H}_{5}\right) .{ }^{31} \mathrm{P}\left\{{ }^{1} \mathrm{H}\right\}-\mathrm{NMR}$ : $\delta_{\mathrm{P}}-84.4(\mathrm{~s}, 5 \%),-158.9(\mathrm{~s}, 14,93 \%),-252.0(\mathrm{~s}, 2 \%) .{ }^{29} \mathrm{Si}\left\{{ }^{1} \mathrm{H}\right\}-$ NMR: $\delta_{\mathrm{Si}}-23.0\left(\mathrm{SiMe}_{2} \mathrm{Ph}\right), 3.42\left(\mathrm{P}\left(\mathrm{SiMe}_{3}\right)_{2}\right)$. Anal. Found: C, 58.29; $\mathrm{H}$, 8.86. Calcd for $\mathrm{C}_{17} \mathrm{H}_{31} \mathrm{PSi}_{3}$ : C, 58.23; $\mathrm{H}, 8.91$.

$\left\{\left(\mathbf{E t}_{2} \mathbf{N}\right)_{2} \mathbf{P}\right\} \mathbf{C}(\mathbf{P h})=\mathbf{C}=\mathbf{C H}_{2} \quad$ (15). To a THF suspension (ca. $30 \mathrm{~cm}^{3}$ ) of excess, pre-activated magnesium turnings containing $\mathrm{HgCl}_{2}\left(0.100 \mathrm{~g}, 3.68 \times 10^{-4} \mathrm{~mol}\right)$ as initiator, was added drop-wise $\mathrm{PhC} \equiv \mathrm{CCH}_{2} \mathrm{Cl}\left(1.00 \mathrm{~g}, 6.65 \times 10^{-3} \mathrm{~mol}\right)$ as solution in THF ( $c a .10 \mathrm{~cm}^{3}$ ); upon complete addition the mixture was brought to reflux for $4 \mathrm{~h}$. After allowing to cool to ambient temperature, the mixture was filtered (via cannula) directly into a pre-cooled $\left(-78{ }^{\circ} \mathrm{C}\right) \mathrm{THF}$ solution of $\left(\mathrm{Et}_{2} \mathrm{~N}\right)_{2} \mathrm{PCl}$ $\left(1.39 \mathrm{~cm}^{3}, 6.65 \times 10^{-3} \mathrm{~mol}\right)$. The resulting red solution was stirred for 30 minutes at this temperature, before allowing it to attain ambient temperature and stir overnight. The resulting orange solution was stripped of volatiles under reduced pressure then extracted with pentane; this fraction was taken to dryness and dried in vacuo to afford the product as dark red oil. Yield: 1.46 g, $76 \% .15$ (74\%): NMR $\left(\mathrm{C}_{6} \mathrm{D}_{6}, 30{ }^{\circ} \mathrm{C}\right):{ }^{1} \mathrm{H}-\mathrm{NMR}$ : $\delta_{\mathrm{H}} 0.89\left(\mathrm{t},{ }^{3} J_{\mathrm{HH}} 7.0 \mathrm{~Hz}, 12 \mathrm{H}, \mathrm{CH}_{3}\right), 3.05\left(\mathrm{q},{ }^{3} J_{\mathrm{HH}} 7.0 \mathrm{~Hz}, 8 \mathrm{H}\right.$, $\left.\mathrm{CH}_{2}\right), 4.69\left(\mathrm{~d}, J_{\mathrm{PH}} 7.0 \mathrm{~Hz}, 2 \mathrm{H},=\mathrm{CH}_{2}\right), 7.11-7.15(\mathrm{~m}, 3 \mathrm{H}, \mathrm{m} / \mathrm{p}-$ $\left.\left.\mathrm{C}_{6} H_{5}\right), 7.63-7.59\left(\mathrm{~m}, 2 \mathrm{H}, o-\mathrm{C}_{6} H_{5}\right) .{ }^{13} \mathrm{C}_{\{}{ }^{1} \mathrm{H}\right\}-\mathrm{NMR}: \delta_{\mathrm{C}} 14.8(\mathrm{~d}$, $\left.\left.{ }^{3} J_{\mathrm{PC}} 3.2 \mathrm{~Hz}, \underline{\mathrm{C}} \mathrm{H}_{3}\right), 43.4\left(\mathrm{~d},{ }^{3} J_{\mathrm{PC}} 17.4 \mathrm{~Hz}, \mathrm{~N} \underline{\mathrm{CH}}\right)_{2}\right), 75.0\left(\mathrm{~s},=\underline{C H}_{2}\right)$, $105.9\left(\mathrm{~d}, J_{\mathrm{PC}} 13.5 \mathrm{~Hz}, \mathrm{i}-\mathrm{C}_{6} \mathrm{H}_{5}\right), 137.4$ (d, $J_{\mathrm{PC}} 19 \mathrm{~Hz}, \mathrm{Ph} \underline{-}-$ $\left\{\mathrm{P}\left(\mathrm{NEt}_{2}\right)_{2}\right\}=\mathrm{C}$ ), $127.8\left(\mathrm{~s}, o-C_{6} \mathrm{H}_{5}\right), 127.9$ (overlapped $m-/ p-C_{6} \mathrm{H}_{5}$ ), $209.9\left(\mathrm{~d}, J_{\mathrm{PC}} 11.4 \mathrm{~Hz},=\underline{C}=\right) .{ }^{31} \mathrm{P}\left\{{ }^{1} \mathrm{H}\right\}-\mathrm{NMR}: \delta_{\mathrm{P}} 91.0(\mathrm{~s}, \mathrm{br}$, $74 \%)$. Propargyl tautomer (5\%): NMR $\left(\mathrm{C}_{6} \mathrm{D}_{6}, 30{ }^{\circ} \mathrm{C}\right):{ }^{1} \mathrm{H}-\mathrm{NMR}$ : $\delta_{\mathrm{H}} 1.02\left(\mathrm{t},{ }^{3} J_{\mathrm{HH}} 7.2 \mathrm{~Hz}, 12 \mathrm{H}, \mathrm{CH}_{3}\right), 2.71\left(\mathrm{~d}, J_{\mathrm{PH}} 5.8 \mathrm{~Hz}, 2 \mathrm{H}\right.$, $\left.\mathrm{CH}_{2} \mathrm{P}\right), 2.87\left(\mathrm{~m}, 8 \mathrm{H}, \mathrm{NCH}_{2}\right) \cdot{ }^{13} \mathrm{C}\left\{{ }^{1} \mathrm{H}\right\}-\mathrm{NMR}: \delta_{\mathrm{C}} 14.0\left(\mathrm{~d},{ }^{3} J_{\mathrm{PC}} 5 \mathrm{~Hz}\right.$, $\left.\underline{C}_{3}\right), 19.8\left(\mathrm{~m}, \underline{C} \mathrm{H}_{2} \mathrm{P}\right), 42.8\left(\mathrm{~d},{ }^{3} J_{\mathrm{PC}} 17 \mathrm{~Hz}, \mathrm{NCH}_{2}\right), 81.5$ (s, $\left.\mathrm{C} \equiv \underline{C} \mathrm{CH}_{2} \mathrm{P}\right), 87.6\left(\mathrm{~s}, \underline{C} \equiv \mathrm{CCH}_{2} \mathrm{P}\right) .{ }^{31} \mathrm{P}\left\{{ }^{1} \mathrm{H}\right\}$-NMR: $\delta_{\mathrm{P}} 83.2$ (s, br, $5 \%)$.

$\left\{\left(\mathbf{E t}_{2} \mathbf{N}\right)(\mathbf{C l}) \mathbf{P}\right\} \mathbf{C}(\mathbf{P h})=\mathbf{C}=\mathbf{C H}_{\mathbf{2}}$ (16). To an ethereal solution of 15 held at $-78{ }^{\circ} \mathrm{C}$ was added drop-wise two equivalent of $\mathrm{HCl}$ (1 $\mathrm{M}$ in ether). The mixture was held at $-78{ }^{\circ} \mathrm{C}$ while stirring for $20 \mathrm{~min}$, before being allowed to warm to ambient temperature and stir overnight. The resulting suspension was filtered and stripped of volatiles under reduced pressure, the resulting orange oil was dried in vacuo. NMR $\left(\mathrm{C}_{6} \mathrm{D}_{6}, 30{ }^{\circ} \mathrm{C}\right):{ }^{1} \mathrm{H}-\mathrm{NMR}$ : $\delta_{\mathrm{H}} 0.81\left(\mathrm{t},{ }^{3} J_{\mathrm{HH}} 6.9 \mathrm{~Hz}, 6 \mathrm{H}, \mathrm{CH}_{3}\right), 2.94\left(\mathrm{q},{ }^{3} J_{\mathrm{HH}} 7.4 \mathrm{~Hz}, 4 \mathrm{H}\right.$, $\left.\mathrm{CH}_{2}\right), 4.89\left(\mathrm{dd},{ }^{2} J_{\mathrm{HH}} 13.0 \mathrm{~Hz}, J_{\mathrm{PH}} 5.7 \mathrm{~Hz}, 1 \mathrm{H},=\mathrm{CH}_{2}\right), 4.93(\mathrm{dd}$, $\left.{ }^{2} J_{\mathrm{HH}} 13.0 \mathrm{~Hz}, J_{\mathrm{PH}} 5.7 \mathrm{~Hz}, 1 \mathrm{H},=\mathrm{CH}_{2}\right), 6.94-7.02(\mathrm{~m}, 1 \mathrm{H}$, $\left.p-\mathrm{C}_{6} \mathrm{H}_{5}\right), 7.11\left(7, J_{\mathrm{HH}} 7.8 \mathrm{~Hz}, 2 \mathrm{H}, m-\mathrm{C}_{6} H_{5}\right), 7.50\left(\mathrm{~d}, J_{\mathrm{HH}} 7.8 \mathrm{~Hz}\right.$, $\left.2 \mathrm{H}, o-\mathrm{C}_{6} H_{5}\right) \cdot{ }^{13} \mathrm{C}\left\{{ }^{1} \mathrm{H}\right\}-\mathrm{NMR}: \delta_{\mathrm{C}} 13.9\left(\mathrm{~d},{ }^{3} J_{\mathrm{PC}} 6.2 \mathrm{~Hz}, \underline{\mathrm{C}} \mathrm{H}_{3}\right), 43.9$ (d, $\left.{ }^{3} J_{\mathrm{PC}} 13 \mathrm{~Hz}, \mathrm{NCH}_{2}\right), 77.6\left(\mathrm{~s},=\underline{\mathrm{C}} \mathrm{H}_{2}\right), 105.3\left(\mathrm{~d}, \bar{J}_{\mathrm{PC}} 40 \mathrm{~Hz}\right.$, $\left.\mathrm{Ph} \underline{\underline{C}}\left\{\mathrm{PCl}\left(\mathrm{NEt}_{2}\right)\right\}=\mathrm{C}\right), 135.4\left(\mathrm{~d}, J_{\mathrm{PC}} 24 \mathrm{~Hz}, \mathrm{i}-\mathrm{C}_{6} \mathrm{H}_{5}\right), 127.6$ (d, $\left.J_{\mathrm{PC}} 1.5 \mathrm{~Hz}, o-C_{6} \mathrm{H}_{5}\right), 127.98\left(\mathrm{~s}, p-C_{6} \mathrm{H}_{5}\right) 128.9\left(\mathrm{~s}, m-C_{6} \mathrm{H}_{5}\right), 210.6$ $\left(\mathrm{d}, J_{\mathrm{PC}} 8.4 \mathrm{~Hz},=\underline{C}=\right) .{ }^{31} \mathrm{P}\left\{{ }^{1} \mathrm{H}\right\}-\mathrm{NMR}: \delta_{\mathrm{P}} 122.0(\mathrm{~s}, \mathrm{br}, 77 \%)$.

\section{Platinum and palladium complexes}

In a typical procedure, to a suspension of the $\left[\mathrm{MCl}_{2}\right]_{n}(\mathrm{M}=\mathrm{Pt}$, Pd) in DCM was added a cooled DCM solution of the respect- 
ive ligand $(8,11$ or 12$)$. The mixture was stirred overnight then stripped of volatiles under reduced pressure to afford the complexes as yellow solids, which were recrystallised from DCM/ether.

cis-[Pt( $\left.\left.\mathbf{P P h}_{2} \mathbf{C H}_{2} \mathbf{C} \equiv \mathbf{C S n B u}_{3}\right)_{2} \mathbf{C l}_{2}\right] \quad$ (17). Yield: $78 \%$. NMR $\left(\mathrm{C}_{6} \mathrm{D}_{6}, 30{ }^{\circ} \mathrm{C}\right):{ }^{1} \mathrm{H}-\mathrm{NMR}: \delta_{\mathrm{H}} 0.81\left(\mathrm{~m}, 12 \mathrm{H}, \mathrm{SnCH}_{2}\right), 0.88(\mathrm{~m}$, $\left.18 \mathrm{H}, \mathrm{CH}_{3}\right), 1.27\left(\mathrm{~m}, 12 \mathrm{H}, \mathrm{CH}_{2}\right), 1.44\left(\mathrm{~m}, 12 \mathrm{H}, \mathrm{CH}_{2}\right), 3.78(\mathrm{~m}$, $\left.J_{\mathrm{PH}} \sim 5 \mathrm{~Hz}, 4 \mathrm{H}, \mathrm{CH}_{2} \mathrm{P}\right), 6.90-7.01\left(\mathrm{~m}, 12 \mathrm{H}, \mathrm{m} / \mathrm{p}-\mathrm{C}_{6} H_{5}\right)$, 7.63-7.77 (m, 8H,o-C $\left.{ }_{6} H_{5}\right) .{ }^{13} \mathrm{C}\left\{{ }^{1} \mathrm{H}\right\}-\mathrm{NMR}: \delta_{\mathrm{C}} 11.1\left(\mathrm{~s}, \underline{\mathrm{C}} \mathrm{H}_{2} \mathrm{Sn}\right.$, $\left.{ }^{1} J_{117 \mathrm{SnC}} 365 \mathrm{~Hz},{ }^{1} J_{119 \mathrm{SnC}} 381 \mathrm{~Hz}\right), 13.9\left(\mathrm{~s}, \underline{C} \mathrm{H}_{3}\right), 23.8\left(\mathrm{~d},{ }^{1} J_{\mathrm{PC}}\right.$ $\left.27 \mathrm{~Hz}, \underline{\mathrm{CH}}_{2} \mathrm{PPh}_{2}\right), 27.4\left(\mathrm{~s}, \underline{\mathrm{C}} \mathrm{H}_{2} \mathrm{CH}_{2} \mathrm{Sn}, J_{117 \mathrm{SnC}} 58.8, J_{119 \mathrm{SnC}}\right.$ $60.7 \mathrm{~Hz}), 29.2\left(\mathrm{~s}, J_{\mathrm{SnC}} 10 \mathrm{~Hz}, \mathrm{CH}_{3} \underline{C H}_{2}\right), 88.7\left(\mathrm{~m}, \underline{C}=\mathrm{CCH}_{2} \mathrm{PPh}_{2}\right.$ ), $104.0\left(\mathrm{~m}, \mathrm{C} \equiv \underline{C} \mathrm{CH}_{2} \mathrm{PPh}_{2}\right), 127.9$ (br, $\left.m-\mathrm{P}\left(C_{6} \mathrm{H}_{5}\right)_{2}\right)$, 129.a (br, i-P $\left.\left(C_{6} \mathrm{H}_{5}\right)_{2}\right), \quad 131.1 \quad\left(\mathrm{~s}, \quad p-\mathrm{P}\left(C_{6} \mathrm{H}_{5}\right)_{2}\right), 134.4 \quad\left(\mathrm{~m}, \quad o-\mathrm{P}\left(C_{6} \mathrm{H}_{5}\right)_{2}\right)$. ${ }^{31} \mathrm{P}\left\{{ }^{1} \mathrm{H}\right\}-\mathrm{NMR}: \quad \delta_{\mathrm{P}} \quad 6.0 \quad\left(\mathrm{~s}, \quad J_{\mathrm{PtP}} 3618 \mathrm{~Hz}\right) .{ }^{119} \mathrm{Sn}\left\{{ }^{1} \mathrm{H}\right\}-\mathrm{NMR}$ : $\delta_{\mathrm{Sn}}-68.2(\mathrm{~m}) .{ }^{195} \mathrm{Pt}\left\{{ }^{1} \mathrm{H}\right\}-\mathrm{NMR}: \delta_{\mathrm{Pt}}-4407$ (t, $\left.J_{\mathrm{PtP}} 3618 \mathrm{~Hz}\right)$. Anal. Found: C, 50.23; H, 5.95. Calcd for $\mathrm{C}_{54} \mathrm{H}_{78} \mathrm{Cl}_{2} \mathrm{P}_{2} \mathrm{PtSn}_{2}$ Si: C, 50.18; H, 6.08.

cis- $\left[\operatorname{Pt}\left(\mathbf{P P h}_{2} \mathbf{C H}_{2} \mathbf{C} \equiv \mathrm{CSiPr}_{3}{ }_{3}\right)_{2} \mathbf{C l}_{2}\right] \quad$ (18). Yield: $86 \%$. NMR $\left(\mathrm{C}_{6} \mathrm{D}_{6}, 30{ }^{\circ} \mathrm{C}\right):{ }^{1} \mathrm{H}-\mathrm{NMR}: \delta_{\mathrm{H}} 0.84$ (sept., $\left.{ }^{3} \mathrm{~J}_{\mathrm{HH}} 7.1 \mathrm{~Hz}, 6 \mathrm{H}, \mathrm{SiCH}\right)$, $0.93\left(\mathrm{~d},{ }^{3} J_{\mathrm{HH}} 7.1 \mathrm{~Hz}, 36 \mathrm{H}, \mathrm{CH}_{3}\right.$ ), 3.87 (d, $J_{\mathrm{PC}} 10.8 \mathrm{~Hz}, 4 \mathrm{H}$, $\left.\mathrm{CH}_{2} \mathrm{P}\right), 6.82-6.88\left(\mathrm{~m}, 8 \mathrm{H}, m-\mathrm{P}\left(\mathrm{C}_{6} H_{5}\right)_{2}\right), 6.91-6.95(\mathrm{~m}, 4 \mathrm{H}$, $p$-P( $\left.\left.\mathrm{C}_{6} H_{5}\right)_{2}\right), \quad 7.51-7.57 \quad\left(\mathrm{~m}, \quad 8 \mathrm{H}, \quad o-\mathrm{P}\left(\mathrm{C}_{6} H_{5}\right)_{2}\right) .{ }^{13} \mathrm{C}\left\{{ }^{1} \mathrm{H}\right\}-\mathrm{NMR}$ : $\delta_{\mathrm{C}} 11.6(\mathrm{~s}, \mathrm{Si} \underline{C} \mathrm{H}), 18.8\left(\mathrm{~s}, \underline{C} \mathrm{H}_{3}\right), 23.9\left(\mathrm{~d}, J_{\mathrm{PC}} 40 \mathrm{~Hz}, \underline{C H}_{2} \mathrm{P}\right), 85.8$ $\left(\mathrm{m}, \underline{C}=\mathrm{CCH}_{2} \mathrm{P}\right), 101.9\left(\overline{\mathrm{m}}, \mathrm{C} \equiv{ }_{C} \mathrm{CH}_{2} \mathrm{P}\right), 127.9\left(\mathrm{~m}, m-C_{6} \mathrm{H}_{5}\right)$, $131.1\left(\mathrm{~s}, p-C_{6} \mathrm{H}_{5}\right), 134.2\left(\mathrm{~m}, o-C_{6} \mathrm{H}_{5}\right), 134.6\left(\mathrm{~m}, \mathrm{i}-C_{6} \mathrm{H}_{5}\right) .{ }^{31} \mathrm{P}\left\{{ }^{1} \mathrm{H}\right\}-$ NMR: $\delta_{\mathrm{P}} 5.83$ (s, $\left.J_{\mathrm{PtP}} 3614 \mathrm{~Hz}\right) .{ }^{29} \mathrm{Si}\left\{{ }^{1} \mathrm{H}\right\}-\mathrm{NMR}: \delta_{\mathrm{Si}}-2.98$. ${ }^{195} \mathrm{Pt}\left\{{ }^{1} \mathrm{H}\right\}-\mathrm{NMR}: \delta_{\mathrm{Pt}}-4399\left(\mathrm{t}, J_{\mathrm{PtP}} 3614 \mathrm{~Hz}\right)$. Anal. Found: C, 56.03; H, 6.39. Calcd for $\mathrm{C}_{48} \mathrm{H}_{66} \mathrm{Cl}_{2} \mathrm{P}_{2} \mathrm{PtSi}_{2}$ : C, 56.13; H, 6.48.

cis-[Pt( $\left.\left.\mathbf{P P h}_{2} \mathbf{C H}_{2} \mathbf{C} \equiv \mathrm{CSiPr}_{3}{ }_{3}\right)_{2} \mathbf{C l}_{2}\right] \quad$ (19). Yield: $78 \%$. NMR $\left(\mathrm{C}_{6} \mathrm{D}_{6}, 30{ }^{\circ} \mathrm{C}\right):{ }^{1} \mathrm{H}-\mathrm{NMR}: \delta_{\mathrm{H}} 0.41\left(\mathrm{~m}, 12 \mathrm{H}, \mathrm{SiCH}_{2}\right), 0.93\left(\mathrm{t},{ }^{3} \mathrm{~J}_{\mathrm{HH}}\right.$ $\left.7.2 \mathrm{~Hz}, 18 \mathrm{H}, \mathrm{CH}_{3}\right), 1.23\left(\mathrm{~m}, 12 \mathrm{H}, \mathrm{CH}_{2} \mathrm{CH}_{2} \mathrm{Si}\right), 3.81$ (d, $J_{\mathrm{PH}}$ $\left.10 \mathrm{~Hz}, 4 \mathrm{H}, \mathrm{CH}_{2} \mathrm{P}\right), 6.86-6.93\left(\mathrm{~m}, 8 \mathrm{H}, m-\mathrm{P}\left(\mathrm{C}_{6} \mathrm{H}_{5}\right)_{2}\right), 6.94-7.00(\mathrm{~m}$, $\left.4 \mathrm{H}, o-\mathrm{P}\left(\mathrm{C}_{6} \mathrm{H}_{5}\right)_{2}\right), 7.54-7.62\left(\mathrm{~m}, 8 \mathrm{H}, o-\mathrm{P}\left(\mathrm{C}_{6} \mathrm{H}_{5}\right)_{2}\right) .{ }^{13} \mathrm{C}\left\{{ }^{1} \mathrm{H}\right\}-\mathrm{NMR}$ : $\delta_{\mathrm{C}} 16.3\left(\mathrm{~s}, \underline{\mathrm{C}} \mathrm{H}_{2} \mathrm{Si},{ }^{1} J_{\mathrm{SiC}} 55 \mathrm{~Hz}\right.$ ), $17.8\left(\mathrm{~s}, \underline{\mathrm{C}} \mathrm{H}_{3}\right), 18.5\left(\mathrm{~s}, \underline{\mathrm{CH}_{2}} \mathrm{CH}_{2} \mathrm{Si}\right.$, ${ }^{2} J_{\mathrm{SiC}} 7.4 \mathrm{~Hz}$ ), 23.9 (d, $\left.J_{\mathrm{PC}} 46 \mathrm{~Hz}, \underline{C} \mathrm{H}_{2} \mathrm{P}\right), 88.0\left(\mathrm{~m}, \underline{C}=\mathrm{CCH}_{2} \mathrm{Cl}\right.$ ), $101.4\left(\mathrm{~m}, \mathrm{C} \equiv \mathrm{CCH}_{2} \mathrm{P}\right), 128.2\left(\mathrm{~m}, m-C_{6} \mathrm{H}_{5}\right), 129.0\left(\mathrm{~s}, p-C_{6} \mathrm{H}_{5}\right)$, $131.1\left(\mathrm{~s}, o-C_{6} \mathrm{H}_{5}\right), 134.3\left(\mathrm{~m}, \mathrm{i}-C_{6} \mathrm{H}_{5}\right) .{ }^{31} \mathrm{P}\left\{{ }^{1} \mathrm{H}\right\}-\mathrm{NMR}: \delta_{\mathrm{P}} 5.95(\mathrm{~s}$, $\left.J_{\mathrm{PtP}} 3614 \mathrm{~Hz}\right) .{ }^{29} \mathrm{Si}\left\{{ }^{1} \mathrm{H}\right\}-\mathrm{NMR}: \delta_{\mathrm{Si}}-13.9 .{ }^{195} \mathrm{Pt}\left\{{ }^{1} \mathrm{H}\right\}-\mathrm{NMR}: \delta_{\mathrm{Pt}}$ -4403 (t, $J_{\mathrm{PtP}} 3614 \mathrm{~Hz}$ ). Anal. Found: C, 56.13; H, 6.45. Calcd for $\mathrm{C}_{48} \mathrm{H}_{66} \mathrm{Cl}_{2} \mathrm{P}_{2} \mathrm{PtSi}_{2}$ : C, 56.13; H, 6.48.

trans $-\left[\mathbf{P d}\left(\mathbf{P P h}_{2} \mathbf{C H}_{2} \mathbf{C} \equiv \mathrm{CSiPr}_{3}{ }_{3}\right)_{2} \mathbf{C l}_{2}\right]$ (20). Yield: $88 \%$. NMR $\left(\mathrm{C}_{6} \mathrm{D}_{6}, 30{ }^{\circ} \mathrm{C}\right):{ }^{1} \mathrm{H}-\mathrm{NMR}: \delta_{\mathrm{H}} 0.90(\mathrm{~m}, 6 \mathrm{H}, \mathrm{SiCH}), 0.97\left(\mathrm{~d},{ }^{3} \mathrm{~J}_{\mathrm{HH}}\right.$ $\left.6.7 \mathrm{~Hz}, 36 \mathrm{H}, \mathrm{CH}_{3}\right), 3.74\left(\mathrm{t}, J_{\mathrm{PH}} 3.9 \mathrm{~Hz}, 2 \mathrm{H}, \mathrm{CH}_{2} \mathrm{P}\right), 7.05-7.11(\mathrm{~m}$, $\left.12 \mathrm{H}, m / p-\mathrm{P}\left(\mathrm{C}_{6} H_{5}\right)_{2}\right), 7.92-7.98\left(\mathrm{~m}, 8 \mathrm{H}, o-\mathrm{P}\left(\mathrm{C}_{6} H_{5}\right)_{2}\right) .{ }^{13} \mathrm{C}\left\{{ }^{1} \mathrm{H}\right\}-$ NMR: $\delta_{\mathrm{C}} 11.6(\mathrm{~s}, \mathrm{Si} \underline{\mathrm{H}}), 18.8\left(\mathrm{~s}, \underline{\mathrm{C}} \mathrm{H}_{3}\right), 18.9$ (t, $J_{\mathrm{PC}} 13.6 \mathrm{~Hz}$, $\left.\underline{\mathrm{CH}_{2}}{ }_{2} \mathrm{P}\right), 85.8\left(\mathrm{~d}, J_{\mathrm{PC}} 2.9 \mathrm{~Hz}, \underline{C}=\mathrm{CCH}_{2} \mathrm{Cl}\right), 101.3\left(\mathrm{~d}, J_{\mathrm{PC}} 5.6 \mathrm{~Hz}\right.$, $\left.\overline{\mathrm{C}} \equiv \underline{C} \mathrm{CH}_{2} \mathrm{P}\right), 128.0\left(\mathrm{~m}, m-C_{6} \overline{\mathrm{H}}_{5}\right), 129.3\left(\mathrm{t}, J_{\mathrm{PC}} 24 \mathrm{~Hz}, \mathrm{i}-C_{6} \mathrm{H}_{5}\right)$, $130 . \overline{9}\left(\mathrm{~s}, p-C_{6} \mathrm{H}_{5}\right), 134.6\left(\mathrm{t}, J_{\mathrm{PC}} 6 \mathrm{~Hz}, o-C_{6} \mathrm{H}_{5}\right) .{ }^{31} \mathrm{P}\left\{{ }^{1} \mathrm{H}\right\}-\mathrm{NMR}$ : $\delta_{\mathrm{P}} 16.0(\mathrm{~s}) .{ }^{29} \mathrm{Si}\left\{{ }^{1} \mathrm{H}\right\}-\mathrm{NMR}: \delta_{\mathrm{Si}}-2.75$. Anal. Found: C, 61.07; $\mathrm{H}$, 6.94. Calcd for $\mathrm{C}_{48} \mathrm{H}_{66} \mathrm{Cl}_{2} \mathrm{P}_{2} \mathrm{PdSi}_{2}$ : C, 61.43; H, 7.09.

trans-[Pd( $\left.\left.\mathbf{P P h}_{2} \mathbf{C H}_{2} \mathbf{C} \equiv \mathrm{CSiPr}_{3}{ }_{3}\right)_{2} \mathbf{C l}_{2}\right]$ (21). Yield: $89 \%$. NMR $\left(\mathrm{C}_{6} \mathrm{D}_{6}, 30{ }^{\circ} \mathrm{C}\right):{ }^{1} \mathrm{H}-\mathrm{NMR}: \delta_{\mathrm{H}} 0.45\left(\mathrm{~m}, 12 \mathrm{H}, \mathrm{SiCH}_{2}\right), 0.91(\mathrm{t}$, $\left.{ }^{3} J_{\mathrm{HH}} 7.0 \mathrm{~Hz}, 18 \mathrm{H}, \mathrm{CH}_{3}\right), 1.25\left(\mathrm{~m}, 12 \mathrm{H}, \mathrm{CH}_{2} \mathrm{CH}_{2} \mathrm{Si}\right), 3.75\left(\mathrm{t}, J_{\mathrm{PH}}\right.$ $\left.4 \mathrm{~Hz}, 4 \mathrm{H}, \mathrm{CH}_{2} \mathrm{P}\right), 7.03-7.12\left(\mathrm{~m}, 12 \mathrm{H}, m / p-\mathrm{P}\left(\mathrm{C}_{6} H_{5}\right)_{2}\right), 7.89-7.98$ $\left(\mathrm{m}, 8 \mathrm{H}, o-\mathrm{P}\left(\mathrm{C}_{6} H_{5}\right)_{2}\right) .{ }^{13} \mathrm{C}\left\{{ }^{1} \mathrm{H}\right\}-\mathrm{NMR}: \delta_{\mathrm{C}} 16.4\left(\mathrm{~s}, \underline{C} \mathrm{H}_{2} \mathrm{Si},{ }^{1} J_{\mathrm{SiC}}\right.$
$57 \mathrm{~Hz}$ ), $17.8\left(\mathrm{~s}, \underline{\mathrm{C}} \mathrm{H}_{3}\right), 18.5\left(\mathrm{~s}, \underline{\mathrm{C}} \mathrm{H}_{2} \mathrm{CH}_{2} \mathrm{Si},{ }^{2} J_{\mathrm{SiC}} 6 \mathrm{~Hz}\right), 18.8$ (t, $J_{\mathrm{PC}} 13.5 \mathrm{~Hz}, \underline{C} \mathrm{H}_{2} \mathrm{P}$ ), 97.9 (d, $J_{\mathrm{PC}} 2.8 \mathrm{~Hz}, \underline{C}=\mathrm{CCH}_{2} \mathrm{Cl}$ ), 100.8 (d, $\left.J_{\mathrm{PC}} 4.9 \mathrm{~Hz}, \mathrm{C} \equiv \mathrm{CCH}_{2} \mathrm{P}\right), 128.2\left(\mathrm{~m}, m-C_{6} \mathrm{H}_{5}\right), 129.2\left(\mathrm{t}, J_{\mathrm{PC}} 24 \mathrm{~Hz}\right.$, $\left.\mathrm{i}-C_{6} \mathrm{H}_{5}\right), 130.9\left(\mathrm{~s}, p-C_{6} \mathrm{H}_{5}\right), 134.7\left(\mathrm{t}, J_{\mathrm{PC}} 5.5 \mathrm{~Hz}, o-C_{6} \mathrm{H}_{5}\right) .{ }^{31} \mathrm{P}\left\{{ }^{1} \mathrm{H}\right\}-$ NMR: $\delta_{\mathrm{P}} 15.9$ (s, $J_{\text {SiP }} 23 \mathrm{~Hz}$ ). ${ }^{29} \mathrm{Si}\left\{{ }^{1} \mathrm{H}\right\}-\mathrm{NMR}: \delta_{\mathrm{Si}}$-13.8. Anal. Found: C, 61.08; $\mathrm{H}, 7.00$. Calcd for $\mathrm{C}_{48} \mathrm{H}_{66} \mathrm{Cl}_{2} \mathrm{P}_{2} \mathrm{PdSi}_{2}$ : C, 61.43; H, 7.09.

cis-/trans-Isomerisation of $\quad\left[\mathrm{Pt}\left(\mathrm{PPh}_{2} \mathrm{CH}_{2} \mathrm{C} \equiv \mathrm{CSiPr}_{3}{ }_{3}\right)_{2} \mathrm{Cl}_{2}\right]$ (19). In a borosilicate NMR tube was placed cis-19 as solution in $\mathrm{C}_{6} \mathrm{D}_{6}$. The sample was irradiated for $20 \mathrm{~min}$. with a $500 \mathrm{~mW}$ full spectrum mercury lamp, resulting in precipitation of an orange solid, which redissolved upon agitation. Yield of trans-19 (by ${ }^{1} \mathrm{H}$ NMR): 58\%. NMR $\left(\mathrm{C}_{6} \mathrm{D}_{6}, 30{ }^{\circ} \mathrm{C}\right):{ }^{1} \mathrm{H}-\mathrm{NMR}$ : $\delta_{\mathrm{H}} 0.46\left(\mathrm{~m}, 12 \mathrm{H}, \mathrm{SiCH}_{2}\right), 0.92\left(\mathrm{t},{ }^{3} \mathrm{~J}_{\mathrm{HH}} 7.3 \mathrm{~Hz}, 18 \mathrm{H}, \mathrm{CH}_{3}\right), 1.25$ $\left(\mathrm{m}, 12 \mathrm{H}, \mathrm{CH}_{2} \mathrm{CH}_{2} \mathrm{Si}\right), 3.77\left(\mathrm{t}, J_{\mathrm{PH}} 4.3 \mathrm{~Hz}, 4 \mathrm{H}, \mathrm{CH}_{2} \mathrm{P}\right), 7.03-7.13$ $\left(\mathrm{m}, 12 \mathrm{H}, \mathrm{m} / p-\mathrm{P}\left(\mathrm{C}_{6} \mathrm{H}_{5}\right)_{2}\right), 7.95-8.01\left(\mathrm{~m}, 8 \mathrm{H}, o-\mathrm{P}\left(\mathrm{C}_{6} H_{5}\right)_{2}\right) .{ }^{13} \mathrm{C}\left\{{ }^{1} \mathrm{H}\right\}-$ NMR: $\delta_{\mathrm{C}} 16.4\left(\mathrm{~s}, \underline{\mathrm{C}} \mathrm{H}_{2} \mathrm{Si}\right), 17.8\left(\mathrm{~s}, \underline{\mathrm{C}} \mathrm{H}_{3}\right), 18.5\left(\mathrm{~s}, \underline{\mathrm{C}} \mathrm{H}_{2} \mathrm{CH}_{2} \mathrm{Si}\right), 23.8$ (t, $\left.J_{\mathrm{PC}} 24 \mathrm{~Hz}, \underline{C} \mathrm{H}_{2} \mathrm{P}\right), 88.0\left(\mathrm{~m}, \underline{C}=\mathrm{CCH}_{2} \mathrm{Cl}\right.$ ), 101.4 (t, $J_{\mathrm{PC}} 6.3 \mathrm{~Hz}$, $\left.\mathrm{C} \equiv \mathrm{CCH}_{2} \mathrm{P}\right), 128.2 \quad\left(\mathrm{~m}, m-C_{6} \mathrm{H}_{5}\right), 128.8 \quad\left(\mathrm{~s}, p-C_{6} \mathrm{H}_{5}\right), 130.9$ $\left(\mathrm{s}, \quad{ }^{o}-C_{6} \mathrm{H}_{5}\right), 134.7$ (t, $J$ PC $\left.6.0 \mathrm{~Hz}, \quad \mathrm{i}-C_{6} \mathrm{H}_{5}\right) .{ }^{31} \mathrm{P}\left\{{ }^{1} \mathrm{H}\right\}-\mathrm{NMR}$ : $\delta_{\mathrm{P}} 11.5\left(\mathrm{~s}, J_{\mathrm{PtP}} 2601 \mathrm{~Hz}\right) .{ }^{29} \mathrm{Si}\left\{{ }^{1} \mathrm{H}\right\}-\mathrm{NMR}: \delta_{\mathrm{Si}}-13.2 .{ }^{195} \mathrm{Pt}\left\{{ }^{1} \mathrm{H}\right\}-$ NMR: $\delta_{\mathrm{Pt}}-3993\left(\mathrm{t}, J_{\mathrm{PtP}} 2601 \mathrm{~Hz}\right)$.

\section{Acknowledgements}

We thank the Royal Society and Leverhulme Trust (F/00 230/ AL, studentship to A.J.S.) for financial support. I.R.C. gratefully acknowledges the award of a Royal Society University Research Fellowship.

\section{Notes and references}

1 For recent reviews see: D. W. Allen, Organophosphorus Chem., 2015, 44, 1-55; D. W. Allen, Organophosphorus Chem., 2014, 43, 1-51 and preceding volumes in the series; D. H. Valentine Jr. and J. H. Hillhouse, Synthesis, 2003, 2437-2460; D. H. Valentine Jr. and J. H. Hillhouse, Synthesis, 2003, 317-334.

2 For a recent review see: E. Bernoud, R. Veillard, C. Alayrac and A.-C. Gaumont, Molecules, 2012, 17, 14573-14587.

3 R. J. Detz, S. A. Heras, R. de Gelder, P. W. N. M. van Leeuwen, H. Hiemstra, J. N. H. Reek and J. H. van Maarseveen, Org. Lett., 2006, 8, 3227-3230.

4 T. D. Simone, R. S. Dickson, B. W. Skelton and A. H. White, Inorg. Chim. Acta, 1995, 240, 323-333.

5 N. Maigrot, M. Melaimi, L. Ricard and P. Le Floch, Heteroatom. Chem., 2003, 14, 326-333.

6 M. Gandelman, E. M. Schuster and G. Nisnevich, US Patent US8318960B2, 2012.

7 M. Reisser, A. Maier and G. Maas, Synlett, 2002, 1459-1462.

8 T. J. Taylor, A. P. Soto, K. Huynh, A. J. Lough, A. C. Swain, N. C. Norman, C. A. Russell and I. Manners, Macromolecules, 2010, 43, 7446-7452.

9 T. W. Mackewitz and M. Regitz, Liebigs Ann., 1996, 327. 
10 R. B. King and A. Efraty, Inorg. Chim. Acta, 1970, 4, 123128.

11 T. W. Mackewitz, D. Ulrich, U. Bergstrasser, S. Leininger and M. Regitz, Liebigs Ann., 1997, 1827-1839.

12 R. Popp, R. Gleiter and F. Rominger, Tetrahedron Lett., 2000, 41, 4075-4078.

13 M. Mirza-Aghayan, R. Boukherroub, G. Oba, G. Manuel and M. Koenig, J. Organomet. Chem., 1998, 564, 61-70.

14 R. H. Shay, B. N. Diel, D. M. Schubert and A. D. Norman, Inorg. Chem., 1988, 27, 2378-2382.

15 T. Baumgartner, Acc. Chem. Res., 2014, 47, 1613-1622; T. Baumgartner and R. Reau, Chem. Rev., 2006, 106, 46814727 and references therein.

16 D. W. Stephan, J. Am. Chem. Soc., 2015, 137, 10018-10032; D. W. Stephan and G. Erker, Angew. Chem., Int. Ed., 2010, 49, 46-76 and references therein.

17 K. Maitra, V. J. Catalano and J. H. Nelson, J. Organomet. Chem., 1997, 529, 409-422.

18 K. Maitra, W. L. Wilson, M. M. Jemin, C. Yeung, W. S. Rader, K. D. Redwine, D. P. Striplin, V. J. Catalano, J. H. Nelson, S. Song and E. C. Alyea, Synth. React. Inorg. Met.-Org. Chem., 1996, 26, 967-996; W. L. Wilson, N. W. Alcock, E. C. Alyea, S. Song and J. H. Nelson, Bull. Soc. Chim. Fr., 1993, 130, 673-682.

19 H. Lang, U. Lay, M. Leise and L. Zsolnai, Z. Naturforsch., B: Chem. Sci., 1993, 48, 27-36.

20 B. N. Diel, P. F. Brandt, R. C. Haltiwanger, M. L. J. Hackney and A. D. Norman, Inorg. Chem., 1989, 28, 2811-2816.

21 R. Regragui and P. H. Dixneuf, New J. Chem., 1988, 12, 547550.

22 J. T. Lin, S. Y. Wang, Y. C. Chou, M. L. Gong, Y.-M. Shiow, H.-M. Gau and Y. S. Wen, J. Organomet. Chem., 1996, 508, 183-193.

23 J.-S. Huang, G.-A. Yu, J. Xie, K.-M. Wong, N. Zhu and C.-M. Che, Inorg. Chem., 2008, 47, 9166-9181.

24 M. Alonso, M. A. Alvarez, E. Garcia, D. Garcia-Vivó and M. A. Ruiz, Inorg. Chem., 2010, 49, 8962-8976.

25 A. I. Arkhypchuk, A. Orthaber, V. A. Mihali, A. Ehlers, K. Lammertsma and S. Ott, Chem. Eur. J., 2013, 19, 1369213704.

26 R. B. King and A. Efraty, Inorg. Chim. Acta, 1970, 4, 123-128.

27 G. Hogarth and J. Y. Pang, J. Organomet. Chem., 1996, 515, 193-203.

28 (a) A. J. Saunders, I. R. Crossley, M. P. Coles and S. M. Roe, Chem. Commun., 2012, 48, 5766-5768; (b) C. E. Averre, M. P. Coles, I. R. Crossley and I. J. Day, Dalton Trans., 2012, 41, 278-284; (c) V. K. Greenacre, M. B. Ansell, S. M. Roe and I. R. Crossley, Eur. J. Inorg. Chem., 2014, 5053-5062.

29 (a) V. K. Greenacre, N. Trathen and I. R. Crossley, Organometallics, 2015, 34, 2533-2542; (b) N. Trathen, M. C. Leech, I. R. Crossley, V. K. Greenacre and S. M. Roe, Dalton Trans.,
2014, 43, 9004-9007; (c) N. Trathen, V. K. Greenacre, I. R. Crossley and S. M. Roe, Organometallics, 2013, 32, 2501-2504.

30 K. Ruitenberg, H. Westmijze, H. Klejn and P. Vermeer, J. Organomet. Chem., 1984, 277, 227-234.

31 K. Kiyokawa, N. Tachikake, M. Yasuda and A. Baba, Angew. Chem., Int. Ed., 2011, 50, 10393-10396.

32 S. K. Woo, L. M. Geary and M. J. Kirsche, Angew. Chem., Int. Ed., 2012, 51, 7830-7834.

33 O. Tsutsumi and K. Nishiguchi, J. Am. Chem. Soc., 1998, 120, 1938-1939; R. L. Phillips, I.-B. Kim, L. M. Tolbert and U. H. F. Bunz, J. Am. Chem. Soc., 2008, 130, 6952-6954.

34 See for example: H. Gilman and S. Rosenberg, J. Org. Chem., 1959, 24, 2063-2064; D. Seyferth and M. A. Weiner, J. Am. Chem. Soc., 1962, 84, 361-364; D. Seyferth and T. Wada, Inorg. Chem., 1962, 1, 78-83; D. Seyferth, L. G. Vaughan and R. Suzuki, J. Organomet. Chem., 1964, 1, 437-448; D. Seyferth and G. B. Womack, Organometallics, 1986, 5, 2360-2370; J. J. Eisch and J. E. Galle, J. Organomet. Chem., 1988, 341, 293-313; A. K. Brisdon, I. R. Crossley, R. G. Pritchard and J. E. Warren, Inorg. Chem., 2002, 41, 4748-4755.

35 J.-P. Quintard and G. Dumartin, J. Organomet. Chem., 1984, 266, 123-138.

36 P. R. Deacon, N. Devylder, M. S. Hill, M. F. Mahon, K. C. Molloy and G. J. Price, J. Organomet. Chem., 2003, 687, 46-56.

37 G. Pattenden and D. Whybrow, Tetrahedron Lett., 1979, 20, 1885-1888; K. V. Baker, J. M. Brown, N. Hughes, A. J. Skarnulis and A. Sexton, J. Org. Chem., 1991, 56, 698703; G. Courtois, M. Harama and L. Miginiac, J. Organomet. Chem., 1980, 198, 1-14.

38 K. S. Dunne, S. E. Lee and V. Gouveneur, J. Organomet. Chem., 2006, 691, 5246-5259.

39 R. B. King and P. M. Sundaram, J. Org. Chem., 1984, 49, 1784-1789.

40 See for example: R. L. Danheiser, Y. M. Choi, M. Menichincheri and E. J. Stoner, J. Org. Chem., 1993, 58, 322-327.

41 B. Németh, B. Khater, T. Veszprémi and J.-C. Guillemin, Dalton Trans., 2009, 3526-3535; J.-C. Guillemin, P. Savignac and J.-M. Denis, Inorg. Chem., 1991, 30, 2170-2173; F. Nief and F. Mathey, Tetrahedron, 1991, 47, 6673-6680; W. Hewertson, I. C. Taylor and S. Trippett, J. Chem. Soc. C, 1970, 1835-1839.

42 M. P. Simonnin and C. Charrier, Org. Magn. Reson., 1969, 1, 27-49.

43 N. Allefeld, M. Grasse, N. Ignat'ev and B. Hoge, Chem. Eur. J., 2014, 20, 8615-8620.

44 H. Burfer and U. Groetze, J. Organomet. Chem., 1968, 12, 451. 